\title{
KPNA4 Serves as A Biomarker For Pancreatic Adnenocarcinoma KPNA4 Serves as A Promising Diagnostic and Prognostic Biomarker For Human Pancreatic Adenocarcinoma
}

\author{
Yuqian Zhou ( $\nabla$ yuqianzhou@csu.edu.cn ) \\ Second Xiangya Hospital of Central South University \\ HongYi Zhu \\ Second Xiangya Hospital of Central South University \\ Yi Chu \\ Second Xiangya Hospital of Central South University \\ Min Luo \\ Second Xiangya Hospital of Central South University \\ WenYu Li \\ Second Xiangya Hospital of Central South University \\ Liang Lvy \\ Second Xiangya Hospital of Central South University \\ JiRong Huo \\ Second Xiangya Hospital of Central South University
}

\section{Research Article}

Keywords: pancreatic adenocarcinoma, KPNA, transcriptional, prognostic, biomarker

Posted Date: February 1st, 2021

DOI: https://doi.org/10.21203/rs.3.rs-151398/v1

License: (c) (i) This work is licensed under a Creative Commons Attribution 4.0 International License. Read Full License 


\section{Abstract}

Objectives: Growing evidence suggests that aberrant expression of Karyopherin alpha (KPNA) has been involved in the tumor progression of various cancer types. However, the differential expression patterns and prognostic value of the seven KPNA subtypes remain to be investigated. Hence the transcriptional and survival data of KPNAs in patients with pancreatic adenocarcinoma (PAAD) were analyzed.

Methods: Transcriptional and survival data related to KPNA expression in patients with PAAD were derived through ONCOMINE, Gene Expression Profiling Interactive Analysis 2, and Human protein atlas databases. The DNA alteration for KPNAs came from The Cancer Genome Atlas and c-BioPortal. The prognostic value analysis was performed with Kaplan-Meier Plotter. Gene functional enrichment analyses were conducted in database LinkedOmics and Metascape.

Results: The mRNA expression levels of KPNA1-4, 6,7 were found significantly upregulated in pancreatic cancer tissues than in normal pancreas tissues, whereas the aberrant expression level of KPNA5 was no more significant in the former than in the latter. KPNA1, 4, and 7 were associated with tumor stage or grade of PAAD. Nevertheless, the obvious association with clinical outcomes was identified only in the aberrant expression of KPNA4. Further gene functional exploration about KPNA4 displayed KPNA4 strongly associated with adherens junction and tumor metastasis in PAAD.

Conclusion: Our findings suggested that KPNA4 might be a potential diagnostic and a new biomarker of prognosis for patients with pancreatic adenocarcinoma.

\section{Introduction}

Pancreatic adenocarcinoma (PAAD) is one of the most lethal human malignancies for still unknown reasons. For the patients with PAAD, their five-year survival rates have remained at $<10 \%$ due to no improvement in early diagnosis and poor responsiveness to most standard therapy $[1,2]$. Though advances of several most common mutations and alterations as the signatures of PAAD, such as KRAS, CDKN2A, SMAD4, and TP53 mutation in PAAD development that has been detected [2], the utility of these research findings in clinical practice still needs time. Hence, exploring the molecular mechanism underlying PAAD and identifying novel biomarkers will promote understanding this disease better and facilitate the development of targeted therapies effective.

Karyopherin protein includes both importins and exportins. Importin consists of two subunits, karyopherin alpha (importin alpha, also known as KPNA) and karyopherin beta (importin beta, also known as KPNB). By binding the nuclear localization sequences (NLS) on cargo protein, importin transports the complex of proteins and KPNB into the nucleus. Seven subtypes of KPNA genes (KPNA1-KPNA7) and twenty-two subtypes of KPNB have been identified in humans. By binding the nuclear localization signal on the cargo protein, all KPNA acts as an adaptor protein between KPNB and cargo protein to translocate them into the nucleus, while only nine members of KPNB are involved in this process. Different KPNA isoforms present 
preferences for particular kinds of nuclear localization signals on cargo molecules, but the boundary may not always be absolute[3].

KPNAs perform the indispensable role of carrying proteins from the cytoplasm into the nucleus, which has been significant during the differentiation and development of animal cells[3]. Recently, their nontransport functions such as gene regulation and cancer pathogenesis in various cancers have been found $[4,5]$. These cancers include breast, brain, colon, gallbladder and prostate cancer, etc. [6-11]. KPNA7 displayed it can facilitate the growth of pancreatic cancer cells and inhibited the autophagy of these cells in vitro [12]. However, the relationship of the majority of KPNAs with the dysregulated mRNA expression, genetic alterations, prognostic value, and further molecular mechanisms in the patients with PAAD have not been completely investigated yet.

In our study, the expression patterns of different KPNAs family members in PAAD were analyzed from DNA, mRNA, and protein levels based on various databases. Furthermore, the prognostic values and potential functions of KPNAs were also explored. We found KPNA4 in patients with PAAD had potential diagnostic and prognostic value, and hence we investigated it further to elucidate the possible mechanism.

\section{Materials And Methods}

\section{TCGA andONCOMINE database Analysis}

The gene expression array datasets of KPNA family members in PAAD in the Cancer Genome Atlas (TCGA) and of ONCOMINE (www.oncomine.org) database [13] were analyzed. The p-value was restricted at 0.05 and the fold change was set at 1.5. The gene rank was restricted to the top $10 \%$ and data type selected mRNA. For each KPNA gene, the comparison between cancer samples and normal control specimen was conducted.

\section{GEPIA2 Analysis}

Gene Expression Profiling Interactive Analysis 2 (GEPIA2, http://gepia2.cancer-pku.cn/\#index) [14] was used to analyze the transcription levels of KPNAs in patients with PAAD. The mRNA expressions of KPNAs in TCGA cancer specimens were compared with those that matched TCGA normal and GTEx pancreatic data. The cut-off of p-value and log2 of fold change was set at 0.01 and 1 , respectively. The correlation analysis of KPNAs and other genes was calculated with the Pearson correlation coefficient.

\section{The Human Protein Atlas (HPA) Analysis}

The HPA (https://www.proteinatlas.org) can allow displaying immunohistochemistry (IHC) staining of consecutive sections of human normal and specific cancer tissues by using two different antibodies: HPA and CAB. KPNA genes in normal tissues and pancreatic adenocarcinoma tissues are evaluated via this database[15]. Based on the immunohistochemical data of patients with or without pancreatic adenocarcinoma from HPA, we further evaluated the protein expression of KPNA genes. 


\section{c-BioPortal Analysis}

The integrative analysis for cancer genomics of the KPNA family was provided by the c-BioPortal database (http://www.cbioportal.org/) [16, 17]. The data of KPNAs came from two studies (TCGA Firehose Legacy and TCGA Pancancer Atlas), and the frequency of gene alterations (amplification, deep deletion, and missense mutations), copy number variance were assessed. Furthermore, the overall, disease-free, progression-free, and disease-specific survival analysis by using c-BioPortal for the KPNA genes with or without alternation were also performed.

\section{UALCAN Analysis}

UALCAN (http://ualcan.path.uab.edu), a website that includes transcriptional and clinical data from different cancer types in The Cancer Genome Atlas (TCGA) [18], was used to compare the mRNA expression with tumor grades in this study. The transcriptional expression panels for KPNA1-7 based on 176 PAAD patients' grades were compared with 4 normal individuals in the TCGA database. Students' ttest was the analyzed method and $\mathrm{p} \otimes 0.05$ was considered statically significant.

\section{The Kaplan-Meier Plotter Analysis}

In this study, the prognostic value of the mRNA expression of KPNA family members in PAAD was tested using the Kaplan-Meier plotter (www.kmplot.com) [19]. Based on the median expression of mRNA (high vs. low expression), the patients with PAAD were divided into two groups. The overall survival (OS), and relapse-free survival (RFS) of these patients were assessed with the hazard ratio and log-rank p-value. The log P-value less than 0.01 was defined as statistically significant.

\section{LinkedOmics Analysis}

To obtain a novel comprehension of the biological effects of the KPNAs, LinkedOmics (http://linkedomics.org), a platform to analyze and compare cancer multi-omics data within and across tumor types [19], was used to perform gene ontology (GO) and Kyoto Encyclopedia of Genes and Genomes (KEGG) analysis. The analysis was based on the mRNA level and histological type of the TCGAPAAD dataset in this online database. Pearson correlation test and Gene set enrichment analysis (GSEA) was used as the statistical method and enrichment analysis respectively. The KEGG pathway analysis is used False Discovery Rate (FDR) as rank criteria (from LinkFinder Result).

\section{Metascape Analysis}

In our study, Metascape (https://metascape.org) [20], a database to provide a comprehensive gene annotation and analysis, was used to perform the functional enrichment and interactome analysis for the KPNA4 associated genes.

\section{Results}




\section{Transcription Levels of KPNAs in Patients with PAAD.}

The transcriptional levels of seven KPNA family members were compared between cancers and those in normal tissue specimen by using ONCOMINE databases (Significant correlations are shown in Figure 1,Table 1).

The analysis revealed that the mRNA expression of KPNA1, KPNA2, KPNA3, KPNA4, and KPNA6 was upregulated in patients with PAAD, KPNA5 was downregulated. No data is available for KPNA7. In Ishikawa's dataset [21], KPNA1 was upregulated in PAAD compared with that in the normal samples, with a fold change of 1.957 and p-value of 0.029 (Table 1). The transcription levels of KPNA2 were significant elevation in patients with PAAD in three datasets (Table 1). In Segara's dataset, KPNA2 was upregulated in PAAD with a fold change of 4.194 and a p-value of 7.03E-6 [22]. In Pei's and Badea's dataset, KPNA2 was upregulated in PAAD compared with that in the normal samples, with a fold change of 2.485 ( $p-$ value $=7.30 \mathrm{E}-5)$ and $2.644(\mathrm{p}$-value=2.16E-8), respectively $[23,24]$. The transcription levels of KPNA3 was significantly higher in patients with PAAD in Segara's datasets[22]. The fold change of mRNA expression of KPNA3 was 2.194 and a p-value of 1.20E-4 (Table 1). The mRNA levels of KPNA4 (fold change $=1.504$ and $p-$ value $=0.034$ ) and KPNA5 (fold change $=1.536$ and $p-$ value $=5.54 \mathrm{E}-4$ ) in patients with PAAD were significantly higher than those in the normal specimen in Ishikawa's and Buchholz's datasets, respectively (Table 1) $[21,25]$. The transcriptional levels of KPNA6 in PAAD (fold change $=1.883$ and $p$-value $=0.017$ ) were significantly different from those in the normal (Table 1) [21].

\begin{tabular}{|c|c|c|c|c|}
\hline Gene & Fold change & p-value & t-test & Source and /Reference \\
\hline KPNA1 & 1.957 & 0.029 & 1.944 & Ishikawa Pancreas[21] \\
\hline \multirow[t]{3}{*}{ KPNA2 } & 4.194 & 7.03E-6 & 6.322 & Segara Pancreas[22] \\
\hline & 2.485 & 7.30E-5 & 4.74 & Pei Pancreas[23] \\
\hline & 2.644 & $2.16 \mathrm{E}-8$ & 6.451 & Badea Pancreas[24] \\
\hline KPNA3 & 2.194 & $1.20 \mathrm{E}-4$ & 4.957 & Segara Pancreas[22] \\
\hline KPNA4 & 1.504 & 0.034 & 1.866 & Ishikawa Pancreas[21] \\
\hline KPNA5* & 1.536 & $5.54 \mathrm{E}-4$ & 4.602 & Buchholz Pancreas[25] \\
\hline KPNA6 & 1.883 & 0.017 & 2.188 & Ishikawa Pancreas[21] \\
\hline KPNA7 & NA & NA & NA & NA \\
\hline
\end{tabular}

NA, not available; * comparison between pancreatic cancer and pancreatic cancer precursor 
In the GEPIA2 database, box plots revealed the expressional comparison between PAAD tissues and matched normal pancreatic tissues of each KPNA family member. Total 179 tumor cases and 171 normal samples were included. The results demonstrated that KPNA1-4 and KPNA6-7 were significantly upregulated in patients with PAAD (Figure 2a-2d, 2f-g). The transcription levels of KPNA5 were higher in patients with PAAD than normal control, but no significant differentiation (Figure 2e).

\section{Translation Levels of KPNAs in Patients with PAAD}

To verify the translational profiles, the immunohistochemistry (IHC) of KPNAs protein expression was compared between PAAD tissues and their counterparts in the Human Protein Atlas platform (HPA) (Figure 3). Representative images of immunohistochemically stained tissues indicated that KPNA1 and KPNA6 proteins were virtually no differential expression in normal pancreas and tumor tissues (Figure 3a, 3f), while KPNA3 was present at high levels in normal tissues (Figure 3c). In contrast, the proteins of KPNA2, KPNA4, KPNA5, KPNA7 were present in both normal and PAAD tissues, however, the expression levels were higher in the tumor sections (Figure 3b,3d, 3e,3g). Taken together, these results indicate that KPNA2, KPNA4, KPNA5, and KPNA7 are both transcriptionally and translationally upregulated in PAAD. KPNA1, KPNA3, KPNA6 are only transcriptionally upregulated in PAAD.

\section{KPNAs Frequently Altered Genes and their association with survival in Patients with PAAD}

A total of 370 patients' data from two sources (TCGA Firehose Legacy and TCGA Pancancer Atlas) was included. Genes of KPNA family members are altered in 34 (9\%) of query patients (Figure 4). The genetic alterations, such as missense mutations, amplification, and deep deletions were found in almost each KPNA members (Figure 4a). However, two or more alterations were detected only in KPNA4 and KPNA5, and the alternation samples were less than $3 \%$ (Figure 4a). Moreover, the Kaplan-Meier estimate displayed that the overall survival (OS), disease-free (DFS), progression-free (PFS), and disease-specific (DSS) survival were not statistically different in pancreatic adenocarcinoma patients with or without alterations of KPNAs (Figure 4b, 4c, 4d, 4e).

\section{The mRNA expression of KPNAs and the Clinicopathological Parameters of Patients with PAAD.}

The correlation between mRNA expression of each KPNA family member with tumor stage was demonstrated with violin plots in the database GEPIA2. The results displayed the distribution of KPNA4, KPNA5, and KPNA7 mRNA expression correlated with tumor stage significantly, whereas KPNA1, KPNA2, KPNA3, and KPNA6 groups were not (Figure $5 \mathrm{a}-\mathrm{h}$ ).

Then, the correlation between transcription of KPNAs and tumor grade of patients with PAAD was analyzed in the UALCAN database. Based on individual PAAD cancer grade, the expression panels for KPNAs are compared between 4 normal individuals and 176 PAAD patients. The mRNA expression of KPNA1, 4, 7 were found to be significantly correlated with the different grades, while other members in the KPNA family did not significantly differ (Figure 6 a-g). Taking all of our results together, the mRNA 
expression levels of KPNA4 and KPNA7 have indicated an association with both cancer stage and pathological grade in PAAD patients.

\section{Prognostic values of KPNA mRNA expression in patients with PAAD}

We further explored the critical efficiency of KPNAs in the survival of patients with pancreatic adenocarcinoma. The survival curves were plotted with data from patients with PAAD included in Kaplan-Meier plotter website. The results revealed all of the significant OS and RFS curves associated with the KPNA family members (Figure 7 a-g OS; i-o RFS). The upregulated mRNA levels of KPNA4 and KPNA5 conferred a worse overall survival of patients with PAAD when the threshold of the log-rank $p$ values was set at 0.01 (Figure 7d and 7e). High levels of KPNA3 and KPNA4 resulted in a worse relapsefree survival of patients with PAAD when the threshold of log-rank $p$ values was set at 0.05 (Figure $7 \mathrm{k}$ and 7l).

Collectively, KPNA4 demonstrated the most obviously prognostic value for the patients than the other KPNAs members (Figure 7h). Then receiver operating characteristic curve (ROC) of KPNA4 is analyzed by $\mathrm{R}$, the area under the curve (AUC) 0.978 implies that high expression of KPNA4 may be a diagnostic biomarker for patients with PAAD (Figure 7p).

The clinical parameters of KPNA4 in patients with PAAD were also analyzed statistically (Table 2). The differential mRNA expression of KPNA4 is significantly associated with tumor T stage and histologic grade. All these results suggest that KPNA4 might be used to predict prognosis in PAAD. 


\section{Table 2. The relationship between the mRNA level of KPNA4 and clinical characteristics for patients with PAAD based on TCGA-PPAD RNAseq-FPKM}

$\begin{array}{lllll}\text { Characteristic } & \begin{array}{l}\text { Low expression of } \\ \text { KPNA4 }\end{array} & \begin{array}{l}\text { High expression of } \\ \text { KPNA4 }\end{array} & p & \text { statistic method } \\ \mathrm{n} & 89 & 89\end{array}$

Gender, n (\%)

0.88

0.02

Chisq.test

$\begin{array}{lll}\text { Female } & 39(21.9 \%) & 41(23 \%) \\ \text { Male } & 50(28.1 \%) & 48(27 \%)\end{array}$

Age, n (\%)

0.764

0.09

Chisq.test

$<=65$

$45(25.3 \%)$

$48(27 \%)$

$>65$

$44(24.7 \%)$

41 (23\%)

Age, mean $\pm \quad 65.43 \pm 10.53$

$64.07 \pm 11.07$

0.403

0.84

T test

SD

T stage, $\mathrm{n}(\%)$

0.041

Fisher.test

$\begin{array}{lll}\text { T1 } & 5(2.8 \%) & 2(1.1 \%) \\ \text { T2 } & 17(9.7 \%) & 7(4 \%) \\ \text { T3 } & 63(35.8 \%) & 79(44.9 \%) \\ \text { T4 } & 2(1.1 \%) & 1(0.6 \%)\end{array}$

$\mathrm{N}$ stage, $\mathrm{n}(\%)$

$0.222 \quad 1.49$

NO 29 (16.8\%)

$21(12.1 \%)$

N1

57 (32.9\%)

$66(38.2 \%)$

M stage, $n(\%)$

0.644

Fisher.test

MO

32 (38.1\%)

$47(56 \%)$

M1

$1(1.2 \%)$

$4(4.8 \%)$

Pathologic stage, $\mathrm{n}(\%)$

0.069

Fisher.test

$\begin{array}{lll}\text { Stage I } & 15(8.6 \%) & 6(3.4 \%) \\ \text { Stage II } & 68(38.9 \%) & 78(44.6 \%) \\ \text { Stage III } & 2(1.1 \%) & 1(0.6 \%) \\ \text { Stage IV } & 1(0.6 \%) & 4(2.3 \%)\end{array}$

Histologic grade, $\mathrm{n}(\%)$

0.048

Fisher.test

G1

$21(11.9 \%)$

$10(5.7 \%)$ 


\begin{tabular}{|lll|}
\hline G2 & $45(25.6 \%)$ & $50(28.4 \%)$ \\
G3 & $20(11.4 \%)$ & $28(15.9 \%)$ \\
G4 & $2(1.1 \%)$ & $0(0 \%)$ \\
\hline
\end{tabular}

\section{Genes Co-expressed with KPNA4 in patients with PAAD}

To find the biological significance of KPNA4 in PAAD, KPNA4 co-expression mode in the PAAD cohort was predicted by the function module of LinkedOmics (Figure 8). Total 19696 genes were revealed a significant correlation with KPNA4. 8941 genes (dark red dots) were displayed significant positive correlations with KPNA4, whereas 10754 genes (dark green dots) were found significant negative correlations (false discovery rate, FDR < 0.01) (Figure 8a). The top 50 significant genes positively and negatively correlated with KPNA4 were shown in the heat map (Figure 8b). The biological process (BP), cellular components (CPs), and molecular functions (MFs) were predicted with Gene Ontology (GO) analysis. The results showed that these correlated genes participate primarily in biological regulation and metabolic process (BP), membrane and nucleus (CPs), protein binding, and ion binding (MFs) (Figure 8c).

Kyoto Encyclopedia of Genes and Genomes (KEGG) pathway analysis with GSEA demonstrated enrichment of these genes is correlated primarily with adherens junction, and focal adhesion. (Figure 8d) (Figure 9).

Adherens junction is one part of cell adhesion junction, the other two parts are desmosomes and tight junction. Each part contains classes of proteins composed of groups of genes or gene families. Three primary molecular families: cadherins, armadillo proteins, and plakins constitute the adherens junction [26]. To a certain extent, the metastasis and epithelial-mesenchymal transition (EMT) of cancer cells are associated with loss of cell adhesion and increase of cellular mobility [26, 27]. Therefore, we explored whether KPNA4 may impact the metastasis of PAAD or not. 19696 genes correlated with KPNA4 and 64 genes involved in cell adhesion junction and EMT were compared and evaluated (Table 3). The genes associated with EMT mainly include SNAIL, SLUG, TWIST, Zeb1-3, and TGFB1. 49 overlap genes were found in these two gene sets (Figure 10). The Venn diagram was created by website tools (http://bioinformatics.psb.ugent.be/webtools/Venn/). 


\begin{tabular}{|c|c|c|c|c|c|c|c|}
\hline 1.Desmosomes & Genes & P Value & $\mathrm{R}$ vlaue & 2.Adherens Junctions & Genes & $P$ Value & R Value \\
\hline \multirow{7}{*}{ (1).Cadherins } & $D S C 1$ & 0.8 & -0.01 & \multirow{2}{*}{ (1).Cadherins } & $C D H 1$ & 0 & 0.72 \\
\hline & $D S C 2$ & 0 & 0.74 & & $\mathrm{CDH} 2$ & $3.6 \mathrm{e}-06$ & 0.23 \\
\hline & $D S C 3$ & $7 \mathrm{e}-11$ & 0.34 & \multirow{4}{*}{ (2).Armadillos } & CTNNA1 & 0 & 0.81 \\
\hline & DSGI & 0.00035 & 0.18 & & CTNNA2 & 0.1 & -0.082 \\
\hline & $D S G 2$ & 0 & 0.70 & & CTNNA3 & $1.8 \mathrm{e}-15$ & 0.38 \\
\hline & $D S G 3$ & $3 e-12$ & 0.34 & & CTNNB1 & 0 & 0.88 \\
\hline & DSGA & $1.9 \mathrm{e}-08$ & 0.28 & \multirow{2}{*}{ (3).Cytolinker } & CTNNDI & 0 & 0.7 \\
\hline \multirow{5}{*}{ (2).Armadillos } & PKP1 & $2.7 e-15$ & 0.38 & & $C T N N D 2$ & $2.7 e-16$ & -0.39 \\
\hline & PKP2 & 0.054 & 0.10 & \multirow{2}{*}{\multicolumn{4}{|c|}{ 3.Tight Junctions }} \\
\hline & PKP3 & 0 & 0.45 & & & & \\
\hline & PKP4 & 0 & 0.73 & \multirow{13}{*}{ (4)-Accessory proteins } & Genes & $P$ Value & $\mathrm{R}$ Value \\
\hline & $J U P$ & 0 & 0.55 & & $J A M 2$ & 0 & 0.59 \\
\hline (3). Plakin & $D S P$ & 0 & 0.54 & & $J A M 3$ & 0 & 0.71 \\
\hline \multirow{2}{*}{\multicolumn{4}{|c|}{ 3.Tight Junctions }} & & MARVELD2 & 0 & 0.63 \\
\hline & & & & & $T J P 1$ & 0 & 0.82 \\
\hline \multirow{2}{*}{ (1).Occludin } & Genes & P Value & R Value & & $T J P 2$ & 0 & 0.73 \\
\hline & $O C L N$ & 0 & 0.46 & & $T J P 3$ & $7.4 \mathrm{e}-0.9$ & 0.28 \\
\hline \multirow{22}{*}{ (2).Claudins } & CLDN1 & 0 & 0.41 & & $z o-1$ & 0 & 0.7 \\
\hline & CLDN2 & $6.1 \mathrm{e}-08$ & 0.27 & & $z O-2$ & NA & NA \\
\hline & CLDN3 & 0.054 & 0.10 & & zo-3 & $5.4 \mathrm{e}-0.5$ & 0.16 \\
\hline & CLDN4 & 0 & 0.40 & & $M U P P 1$ & 0 & 0.82 \\
\hline & CLDNS & 0.58 & 0.02 & & $M A G I-1$ & 0.037 & -0.081 \\
\hline & CLDN6 & $2.4 \mathrm{e}-06$ & -0.18 & & $C D{ }^{4}$ & 0 & 0.72 \\
\hline & $C L D N 7$ & 0 & 0.33 & & \multirow{2}{*}{\multicolumn{3}{|c|}{ EMT }} \\
\hline & CLDN8 & 0 & 0.47 & & & & \\
\hline & CLDN9 & 0.0053 & 0.15 & & Genes & P Value & $\mathrm{R}$ Value \\
\hline & CLDN10 & 0.03 & 0.12 & & SNAIL & 0 & 0.47 \\
\hline & CLDN11 & 0 & 0.48 & & SLUG & 0 & 0.64 \\
\hline & CLDN12 & 0 & 0.67 & & TWIST & $6.6 \mathrm{e}-08$ & 0.27 \\
\hline & CLDN13 & $\mathrm{NA}$ & $\mathrm{NA}$ & & $Z_{e B 1}$ & 0 & 0.65 \\
\hline & CLDN14 & $1.3 \mathrm{e}-06$ & -0.19 & & $\mathrm{ZeB2}$ & 0 & 0.59 \\
\hline & CLDN15 & 0.12 & 0.06 & & $Z_{e B 3}$ & NA & $\mathrm{NA}$ \\
\hline & CLDN16 & 0.13 & -0.058 & & TGFBI & 0 & 0.37 \\
\hline & CLDN17 & 0.88 & 0.01 & & & & \\
\hline & CLDN18 & 0.64 & -0.018 & \multicolumn{4}{|c|}{ Pearson Correlation Coefficient } \\
\hline & CLDN19 & $1.4 \mathrm{e}-0.8$ & 0.22 & $\mathrm{R}$ Value & \multicolumn{2}{|c|}{ interpretation } & Cell Color \\
\hline & CLDN20 & 0.17 & 0.05 & $0.8-1.0$ & \multicolumn{2}{|c|}{ extremely strong association } & \\
\hline & CLDN21 & $\mathrm{NA}$ & $\mathrm{NA}$ & $0.6-0.8$ & \multicolumn{2}{|c|}{ strong association } & \\
\hline & CLDN22 & $3.2 \mathrm{e}-11$ & -0.25 & $0.4-0.6$ & \multicolumn{2}{|c|}{ middle association } & \\
\hline
\end{tabular}

Correlation Analysis between KPNA4 and genes associated with cell adhesion junction in Patients With PAAD

The overlap 49 genes associated with cell adhesion and EMT were explored in GEPIA2 further (Table 3 and Figure 11). Pearson correlation coefficient more than 0.2 and $p$-value less than 0.01 were set as the threshold, respectively. 43 genes were demonstrated as a linear association with KPNA4. CTNNA1, CTNNB1, TJP1, and MUPP1 were showed an extremely strong correlation with KPNA4 (Table 3 and Figure 
11a). CTNNA1 and CTNNB1 belong to the adherens junction cluster. The plot is based on TCGA PAAD tumor, TCGA PAAD normal and GTX pancreas dataset.

Further network investigation was conducted between KPNA4 and those linear correlated genes in Metascape. The process and pathway enrichment analysis, protein interaction network of associated genes with KPNA4 were displayed in figure $11 \mathrm{~b}$ and $11 \mathrm{c}$. These genes are primarily related to the cell-cell junction organization. The pathway enrichment demonstrated that these genes significantly enriched in pathway neoplastic cell transformation and neoplasm invasiveness (Figure 11d). These results suggested that abnormal expression of KPNA4 may have a major effect on the metastasis of PAAD.

\section{Discussion}

KPNAs are not only passive transporter for proteins, their correlations with different types of cancers are observed recently. Many studies have demonstrated that KPNAs appears to be important in cancer cell growth, migration, and invasion $[9,10]$. More specially, compared with corresponding normal tissues, KPNAs have relatively higher expression levels in various tumors [10, 11, 28-30]. However, the relationship between the elevation of distinct KPNA family members and the exact roles in PAAD is not yet clear. Our study is the first time to perform a deeper analysis of KPNAs in PAAD. We hope our findings will extend the knowledge of pathogenesis and progression for patients with PAAD.

The diseases associated with KPNA1 are mainly virus infection, neuronal damage, and inflammation[31]. Recent studies found it can help nucleocytoplasmic translocation of acetyl-CoA synthetase 2 (ACSS2) to promote glioma tumorigenesis[6, 32]. Also, phosphorylation of E47 accumulating in the nucleus in a KPNA1 dependent manner can facilitate epithelial-mesenchymal transition and metastasis of colon cancer[9]. However, KPNA1 is only an assistant and no direct effect on these cancers. In our report, the mRNA expression of KPNA1 was higher in human PAAD than in normal tissues. However, this elevation was not coincident between transcription and translation level. Compared with normal controls, KPNA1 protein in tumor tissue showed no obvious elevation with immunohistochemical staining. Although the upregulation of KPNA1 correlates with histological grades of the patients with PAAD, high KPNA1 is no correlation with the clinical stages. An increased expression of KPNA1 was significantly associated with poor OS in all of the patients followed up for 80 months, but the RFS was of no significance. All these findings suggest that KPNA1 is not an appropriate prognostic biomarker for PAAD.

As a member of the most research in the KPNA family, KPNA2 was found overexpression in various cancers, which is strongly associated with poor prognosis. The elevation of KPNA2 can promote the development of gallbladder and lung cancer $[10,28]$. USP1-mediated deubiquitination and stabilization of KPNA2 are indispensable for metastasis in breast cancer[8]. KPNA2 is also involved in the survival and metastasis of prostate cancer[33], its upregulation promotes metabolic abnormalities in glioblastomas by controlling c-myc [34]. In our study, KPNA2 was highly expressed on both transcription and translation levels. However, this aberrant upregulation is of no significance for tumor stages and grades in patients with PAAD. The prognostic value of KPNA2 is not obvious either. 
Like KPNA1, KPNA3 has also a strong association with virus infection and brain damage in the previous studies [35]. However, increased expression of KPNA3 was revealed in colorectal cancer and hepatocellular cancer, and that could promote tumors to a high proliferation rate, invasion, and chemotherapy resistance $[29,30]$. In our study, KPNA3 displayed upregulation mRNA level whereas downregulation protein level in tumor tissues. Neither tumor stages nor histological grades are significant in correlation with high KPNA3 in patients with PAAD. There is no obvious correlation between high KPNA3 and prognosis for these patients.

Presently, no studies are exhibited a direct association between KPNA5 or KPNA6 and cancers. KPNA5 and KPNA6 are more related to virus infection. In our report, ONCOMINE datasets and TCGA datasets displayed paradoxical findings of mRNA expression of KPNA5 in tumor tissues, while the results about high KPNA6 expression is consistent in both mentioned datasets. Neither KPNA5 nor KPNA6 showed a marked correlation with tumor stage and grade in patients with PAAD. However, an increased KPNA5 expression showed a significant correlation with poor OS in these patients. Hence, these contradictory findings suggest KPNA5 and KPNA6 are not suitable as a tumor biomarker.

KPNA7 is the only studied member of the KPNA family in pancreatic cancer presently. Overexpressed KPNA7 promotes proliferation of pancreatic cancer cells in vitro[12,36], depletion of KPNA7 can lead to a dramatic reduction in cell growth of pancreatic and breast cancer[37]. In our study, although KPNA7 displayed a marked correlation with tumor stage and grade in patients with PAAD, its elevation is not significantly different with both poor OS and RFS.

KPNA4 plays a crucial role in infection and inflammation diseases too. However, its upregulation contributed to the development and progression of prostate cancer. Inhibition of KPNA4 expression of prostate cancer can suppress this process both in vivo and in vitro [11]. The elevation of KPNA4 mRNA expression is also found in the head and neck of squamous cell carcinoma (HNSCC). After the knockdown of KPNA4, the nuclear localization signal-dependent transport activity was attenuated and therefore the malignant phenotypes of HNSCC were inhibited [38]. The elevation of KPNA4 in other cancers such as papillary thyroid cancer and lung cancer is mediated by microRNA, targeting this miRNA/KPNA4 axis can help to inhibit malignant phenotypes of these cancers $[39,40]$.

In our study, the elevation of KPNA4 appears in many cases of PAAD. Therefore, as a potential diagnostic and prognostic marker, it might be worthwhile to investigate further clinically. Our results suggest the high expression of KPNA4 in tumor tissues occurred on both mRNA and protein levels, and a significant correlation was found between this expression with tumor stage and grade in the patients of PAAD. Besides, we clarified that upregulation of KPNA4 was significantly correlated with not only poor OS, but also poor RFS in all of these patients. Furthermore, analysis with ROC supports KPNA4 might be a biomarker for patients with PAAD.

To explore the molecular mechanisms in regulating abnormal KPNA4 expression, we analyzed the KPNA4 co-expression network. We found that the functional consequence of KPNA4 is primarily involved in adherens junction and focal adhesion. The constitution of the cell adhesive junctions includes adherens 
junction, desmosomes, and tight junction. The function of cell adhesive junctions includes maintenance of the integrity of epithelial cells and regulation of signal communication between cells[26]. The downregulation of cell adhesion molecules is necessary for epithelial-mesenchymal transition (EMT), a process that facilitates the invasion and metastasis of cancer[26, 27]. For mining regulators potentially responsible for KPNA4 upregulation, following protein-protein interaction analysis was performed with these genes associated with adherens junction and EMT. The results demonstrated CTNNA1(E-Cadherin) and CTNNB1(beta-catenin) was an extremely strong correlation with KPNA4 in both GEPIA2 and Metascape databases. Many studies have revealed the pivotal impact of E-Cadherin and beta-catenin on the progression and invasion of tumors. Future studies worth investigating the detailed mechanism between distinct KPNA4 and these molecules in PAAD.

\section{Conclusions}

In this study, our results implied that the increased expression of KPNA4 in pancreatic adenocarcinoma tissues might serve as a promising diagnostic and prognostic biomarker for PAAD. Although our study has some contributions, there are still several limitations. Firstly, all the analyzed experimental data were taken from online databases. Second, the patient numbers of pathologic stage III and IV are less than 10, which might lead to bias in statistics. To verify our finding, additional larger clinical samples are needed.

\section{Declarations}

\section{Ethics Approval and consent to participate}

This study was approved by the Academic Committee of the $2^{\text {nd }}$ XiangYa Hospital. All the data were obtained from the online websites and published literatures, it was confirmed that all written informed consent was obtained. The study was performed according to the principles expressed in the Declaration of Helsinki.

\section{Consent for publication}

Publication consent was obtained from all authors.

\section{AUTHOR CONTRIBUTIONS}

Yuqian Zhou and Jirong Huo conducted and designed the research; Hongyi Zhu wrote the manuscript; Yi Chu, Min Luo, Wenyu Li, and Liang Lyu analyzed data and prepared figures.

\section{Availability of data and material}

All data in our study derived from online databases and is publicly available.

\section{CONFLICTS OF INTEREST}


The authors declare no competing interest.

\section{FUNDING}

This study is funding by Hunan Provincial Natural Science Foundation to Hong Yi Zhu (Grant No. 2019JJ50868), and Hunan Provincial Natural Science Foundation to Yi Chu (Grant No. 2018JJ3720).

\section{Acknowledgements}

Not applicable.

\section{Authors' information}

Hongyi Zhu, M.D.,Yi Chu, M.D., Min Luo, M.D., Wenyu Li, M.D., Liang Lyu, M.D., Jirong Huo, M.D., and Yuqian Zhou, M.D.*

Department of Gastroenterology, the $2^{\text {nd }}$ Xiangya hospital of central south university, Changsha, Hunan, 410010, China

\section{*Corresponding Author}

* Yu-qian Zhou, ORCID Identifier, 0000-0002-8168-3952

Email: yuqianzhou@csu.edu.cn;

Department of Gastroenterology, the $2^{\text {nd }}$ Xiangya hospital of central south university, Changsha, Hunan,410010, China,

+86-0731-85295035;

No. 139 Renmin road, center of digestive endoscopy of the 2nd Xiangya hospital, Changsha, Hunan,410010, China

\section{References}

1. Xiong, G., C. Liu, G. Yang, M. Feng, J. Xu, F. Zhao, et al., Long noncoding RNA GSTM3TV2 upregulates LAT2 and OLR1 by competitively sponging let-7 to promote gemcitabine resistance in pancreatic cancer. J Hematol Oncol, 2019. 12(1): p. 97.

2. Kamisawa, T., L.D. Wood, T. Itoi and K. Takaori, Pancreatic cancer. Lancet, 2016. 388(10039): p. 7385.

3. Goldfarb, D.S., A.H. Corbett, D.A. Mason, M.T. Harreman and S.A. Adam, Importin alpha: a multipurpose nuclear-transport receptor. Trends Cell Biol, 2004. 14(9): p. 505-14.

4. Miyamoto, Y., K. Yamada and Y. Yoneda, Importin alpha: a key molecule in nuclear transport and nontransport functions. J Biochem, 2016. 160(2): p. 69-75. 
5. Mahipal, A. and M. Malafa, Importins and exportins as therapeutic targets in cancer. Pharmacol Ther, 2016. 164: p. 135-43.

6. Li, X., W. Yu, X. Qian, Y. Xia, Y. Zheng, J.H. Lee, et al., Nucleus-Translocated ACSS2 Promotes Gene Transcription for Lysosomal Biogenesis and Autophagy. Mol Cell, 2017. 66(5): p. 684-697 e9.

7. Lu, Y., L. Xiao, Y. Liu, H. Wang, H. Li, Q. Zhou, et al., MIR517C inhibits autophagy and the epithelial-tomesenchymal (-like) transition phenotype in human glioblastoma through KPNA2-dependent disruption of TP53 nuclear translocation. Autophagy, 2015. 11(12): p. 2213-32.

8. Ma, A., M. Tang, L. Zhang, B. Wang, Z. Yang, Y. Liu, et al., USP1 inhibition destabilizes KPNA2 and suppresses breast cancer metastasis. Oncogene, 2019. 38(13): p. 2405-2419.

9. Zhu, G., X. Li, B. Guo, Q. Ke, M. Dong, and F. Li, PAK5-mediated E47 phosphorylation promotes epithelial-mesenchymal transition and metastasis of colon cancer. Oncogene, 2016. 35(15): p. 194354.

10. Xiang, S., Z. Wang, Y. Ye, F. Zhang, H. Li, Y. Yang, et al., E2F1 and E2F7 differentially regulate KPNA2 to promote the development of gallbladder cancer. Oncogene, 2019. 38(8): p. 1269-1281.

11. Yang, J., C. Lu, J. Wei, Y. Guo, W. Liu, L. Luo, et al., Inhibition of KPNA4 attenuates prostate cancer metastasis. Oncogene, 2017. 36(20): p. 2868-2878.

12. Laurila, E., E. Vuorinen, K. Savinainen, H. Rauhala and A. Kallioniemi, KPNA7, a nuclear transport receptor, promotes malignant properties of pancreatic cancer cells in vitro. Exp Cell Res, 2014. 322(1): p. 159-67.

13. Rhodes, D.R., J. Yu, K. Shanker, N. Deshpande, R. Varambally, D. Ghosh, et al., ONCOMINE: a cancer microarray database and integrated data-mining platform. Neoplasia, 2004. 6(1): p. 1-6.

14. Tang, Z., B. Kang, C. Li, T. Chen and Z. Zhang, GEPIA2: an enhanced web server for large-scale expression profiling and interactive analysis. Nucleic Acids Res, 2019. 47(W1): p. W556-W560.

15. Uhlen, M., C. Zhang, S. Lee, E. Sjostedt, L. Fagerberg, G. Bidkhori, et al., A pathology atlas of the human cancer transcriptome. Science, 2017. 357(6352).

16. Cerami, E., J. Gao, U. Dogrusoz, B.E. Gross, S.O. Sumer, B.A. Aksoy, et al., The cBio cancer genomics portal: an open platform for exploring multidimensional cancer genomics data. Cancer Discov, 2012. 2(5): p. 401-4.

17. Gao, J., B.A. Aksoy, U. Dogrusoz, G. Dresdner, B. Gross, S.O. Sumer, et al., Integrative analysis of complex cancer genomics and clinical profiles using the cBioPortal. Sci Signal, 2013. 6(269): p. pl1.

18. Chandrashekar, D.S., B. Bashel, S.A.H. Balasubramanya, C.J. Creighton, I. Ponce-Rodriguez, B. Chakravarthi, et al., UALCAN: A Portal for Facilitating Tumor Subgroup Gene Expression and Survival Analyses. Neoplasia, 2017. 19(8): p. 649-658.

19. Vasaikar, S.V., P. Straub, J. Wang and B. Zhang, LinkedOmics: analyzing multi-omics data within and across 32 cancer types. Nucleic Acids Res, 2018. 46(D1): p. D956-D963.

20. Zhou, Y., B. Zhou, L. Pache, M. Chang, A.H. Khodabakhshi, O. Tanaseichuk, et al., Metascape provides a biologist-oriented resource for the analysis of systems-level datasets. Nat Commun, 2019. 10(1): p. 
1523.

21. Ishikawa, M., K. Yoshida, Y. Yamashita, J. Ota, S. Takada, H. Kisanuki, et al., Experimental trial for diagnosis of pancreatic ductal carcinoma based on gene expression profiles of pancreatic ductal cells. Cancer Sci, 2005. 96(7): p. 387-93.

22. Segara, D., A.V. Biankin, J.G. Kench, C.C. Langusch, A.C. Dawson, D.A. Skalicky, et al., Expression of HOXB2, a retinoic acid signaling target in pancreatic cancer and pancreatic intraepithelial neoplasia. Clin Cancer Res, 2005. 11(9): p. 3587-96.

23. Pei, H., L. Li, B.L. Fridley, G.D. Jenkins, K.R. Kalari, W. Lingle, et al., FKBP51 affects cancer cell response to chemotherapy by negatively regulating Akt. Cancer Cell, 2009. 16(3): p. 259-66.

24. Badea, L., V. Herlea, S.O. Dima, T. Dumitrascu and I. Popescu, Combined gene expression analysis of whole-tissue and microdissected pancreatic ductal adenocarcinoma identifies genes specifically overexpressed in tumor epithelia. Hepatogastroenterology, 2008. 55(88): p. 2016-27.

25. Buchholz, M., M. Braun, A. Heidenblut, H.A. Kestler, G. Kloppel, W. Schmiegel, et al., Transcriptome analysis of microdissected pancreatic intraepithelial neoplastic lesions. Oncogene, 2005. 24(44): p. 6626-36.

26. Knights, A.J., A.P. Funnell, M. Crossley and R.C. Pearson, Holding Tight: Cell Junctions and Cancer Spread. Trends Cancer Res, 2012. 8: p. 61-69.

27. Kyuno, D., A. Takasawa, S. Kikuchi, I. Takemasa, M. Osanai, and T. Kojima, Role of tight junctions in the epithelial-to-mesenchymal transition of cancer cells. Biochim Biophys Acta Biomembr, 2020: p. 183503.

28. Li, X.L., L.L. Jia, M.M. Shi, X. Li, Z.H. Li, H.F. Li, et al., Downregulation of KPNA2 in non-small-cell lung cancer is associated with Oct4 expression. J Transl Med, 2013. 11: p. 232.

29. Liu, T., Z. Han, H. Li, Y. Zhu, Z. Sun, and A. Zhu, LncRNA DLEU1 contributes to colorectal cancer progression via activation of KPNA3. Mol Cancer, 2018. 17(1): p. 118.

30. Hu, B., J.W. Cheng, J.W. Hu, H. Li, X.L. Ma, W.G. Tang, et al., KPNA3 Confers Sorafenib Resistance to Advanced Hepatocellular Carcinoma via TWIST Regulated Epithelial-Mesenchymal Transition. J Cancer, 2019. 10(17): p. 3914-3925.

31. Panayotis, N., A. Sheinin, S.Y. Dagan, M.M. Tsoory, F. Rother, M. Vadhvani, et al., Importin alpha5 Regulates Anxiety through MeCP2 and Sphingosine Kinase 1. Cell Rep, 2018. 25(11): p. 3169-3179 e7.

32. Sang, Y., Y. Li, Y. Zhang, A.A. Alvarez, B. Yu, W. Zhang, et al., CDK5-dependent phosphorylation and nuclear translocation of TRIM59 promotes macroH2A1 ubiquitination and tumorigenicity. Nat Commun, 2019. 10(1): p. 4013.

33. D'Antonio, A., A. Caputo, F. Fraggetta, P. Pepe, L. Insabato, E. Barra, et al., KPNA2/ERG Coexpression is Associated With Early Recurrence in Advanced Prostate Cancers. Appl Immunohistochem Mol Morphol, 2020. 28(1): p. 62-66.

34. Li, J., Q. Liu, Z. Liu, Q. Xia, Z. Zhang, R. Zhang, et al., KPNA2 promotes metabolic reprogramming in glioblastomas by regulation of c-myc. J Exp Clin Cancer Res, 2018. 37(1): p. 194. 
35. Sowa, A.S., E. Martin, I.M. Martins, J. Schmidt, R. Depping, J.J. Weber, et al., Karyopherin alpha-3 is a key protein in the pathogenesis of spinocerebellar ataxia type 3 controlling the nuclear localization of ataxin-3. Proc Natl Acad Sci U S A, 2018. 115(11): p. E2624-E2633.

36. Vuorinen, E.M., N.K. Rajala, H.E. Rauhala, A.T. Nurminen, V.P. Hytönen, and A. Kallioniemi, Search for KPNA7 cargo proteins in human cells reveals MVP and ZNF414 as novel regulators of cancer cell growth. Biochim Biophys Acta Mol Basis Dis, 2017. 1863(1): p. 211-219.

37. Vuorinen, E.M., N.K. Rajala, T.O. Ihalainen and A. Kallioniemi, Depletion of nuclear import protein karyopherin alpha 7 (KPNA7) induces mitotic defects and deformation of nuclei in cancer cells. BMC Cancer, 2018. 18(1): p. 325.

38. Hazawa, M., K. Sakai, A. Kobayashi, H. Yoshino, Y. Iga, Y. Iwashima, et al., Disease-specific alteration of karyopherin-alpha subtype establishes feed-forward oncogenic signaling in head and neck squamous cell carcinoma. Oncogene, 2020. 39(10): p. 2212-2223.

39. Li, X., M. Yu and C. Yang, YY1-mediated overexpression of long noncoding RNA MCM3AP-AS1 accelerates angiogenesis and progression in lung cancer by targeting miR-340-5p/KPNA4 axis. J Cell Biochem, 2020. 121(3): p. 2258-2267.

40. Feng, L., R. Wang, Y. Yang, X. Shen, Q. Shi, M. Lian, et al., KPNA4 regulated by miR-548b-3p promotes the malignant phenotypes of papillary thyroid cancer. Life Sci, 2020: p. 118743.

\section{Figures}




\begin{tabular}{|c|c|c|c|c|c|c|c|c|c|c|c|c|c|}
\hline Analysis Type by Cancer & \multicolumn{2}{|c|}{$\begin{array}{c}\text { Cancer } \\
\text { vs. } \\
\text { Normal }\end{array}$} & \multicolumn{2}{|c|}{$\begin{array}{c}\text { Cancer } \\
\text { vs. } \\
\text { Normal }\end{array}$} & \multicolumn{2}{|c|}{$\begin{array}{c}\text { Cancer } \\
\text { vs. } \\
\text { Normat. }\end{array}$} & \multicolumn{2}{|c|}{$\begin{array}{c}\text { Cancer } \\
\text { vs. } \\
\text { Normat }\end{array}$} & \multicolumn{2}{|c|}{$\begin{array}{c}\text { Cancer } \\
\text { vs. } \\
\text { Normal }\end{array}$} & \multicolumn{2}{|c|}{$\begin{array}{c}\text { Cancer } \\
\text { vs. } \\
\text { Normal }\end{array}$} & $\begin{array}{c}\text { Cancer } \\
\text { vs. } \\
\text { Normal }\end{array}$ \\
\hline Bladder Cancer & & 2 & 7 & & 2 & 2 & 1 & & & & & & \\
\hline Brain and CNS Cancer & & 2 & 6 & 1 & & 4 & 1 & & 3 & & 1 & 2 & \\
\hline Breast Cancer & 9 & 1 & 29 & 1 & & & 6 & 4 & 1 & & 1 & 1 & \\
\hline Cervical Cancer & 2 & & 3 & & & & 2 & & & & & & \\
\hline Colorectal Cancer & 1 & 1 & 15 & & 9 & & 1 & & & & & & \\
\hline Esophageal Cancer & 1 & 1 & 4 & & 1 & & 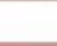 & 1 & & & & & \\
\hline Gastric Cancer & 1 & & 11 & & 1 & & 3 & & & & 3 & & \\
\hline Head and Neck Cancer & 2 & & 13 & & 1 & & 5 & & & & 1 & & \\
\hline Kidney Cancer & 4 & & 4 & & 1 & & 2 & & & & 1 & & \\
\hline Leukemia & & 4 & 1 & 2 & & 3 & 1 & 1 & 2 & 1 & & 1 & \\
\hline Liver Cancer & & 2 & 6 & & & & 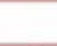 & & & & & & \\
\hline Lung Cancer & 9 & . & 16 & & & & 2 & & 1 & & 1 & & \\
\hline Lymphoma & & 6. & 1 & 1 & & 4 & 1 & & & 3 & 2 & 3 & \\
\hline Melanoma & & & 2 & & 1 & 2 & 2 & 1 & & & 1 & 1 & \\
\hline Myeloma & 2 & & 2 & & & & 1 & & & & & & \\
\hline Other Cancer & 5 & 1 & 8 & 6 & 2 & 6 & 1 & is & & 4 & & 1 & \\
\hline Ovarian Cancer & 1 & & 7 & & & & & & & 3 & & & \\
\hline Pancreatic Cancer & 1 & & 3 & & 1 & & 1 & & & 1 & 1 & & \\
\hline Prostate Cancer & & & 1 & & & 4 & 1 & 1 & & & & 1 & \\
\hline Sarcoma & 3 & 1 & 9 & & 1 & 2 & 2 & & 4 & & & 3 & \\
\hline Significant Unique Analyses & 41 & 20 & 146 & 11 & 20 & 28 & 32 & 14 & 11 & 12 & 12 & 13 & \\
\hline Total Unique Analyses & \multicolumn{2}{|c|}{345} & \multicolumn{2}{|c|}{351} & \multicolumn{2}{|c|}{364} & \multicolumn{2}{|c|}{319} & \multicolumn{2}{|c|}{312} & \multicolumn{2}{|c|}{327} & 0 \\
\hline
\end{tabular}

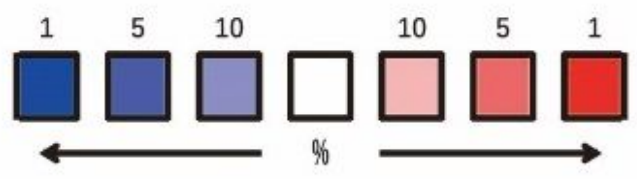

Figure 1

The transcriptional changes of KPNAs expression in patients with PAAD. 


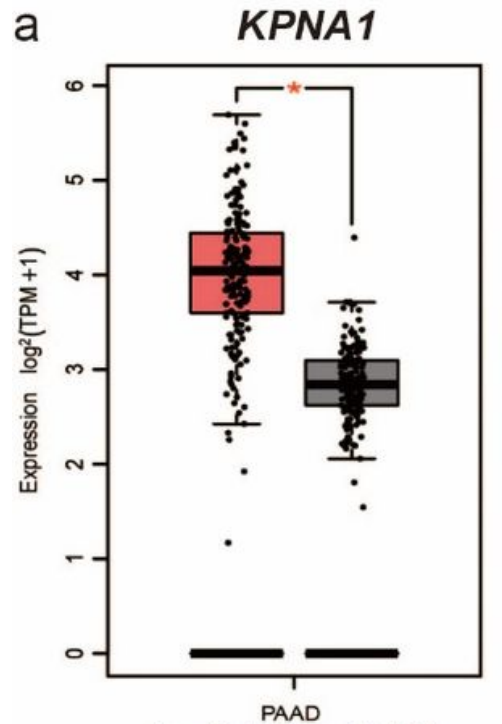

(num(T)=179; num $(\mathrm{N})=171)$

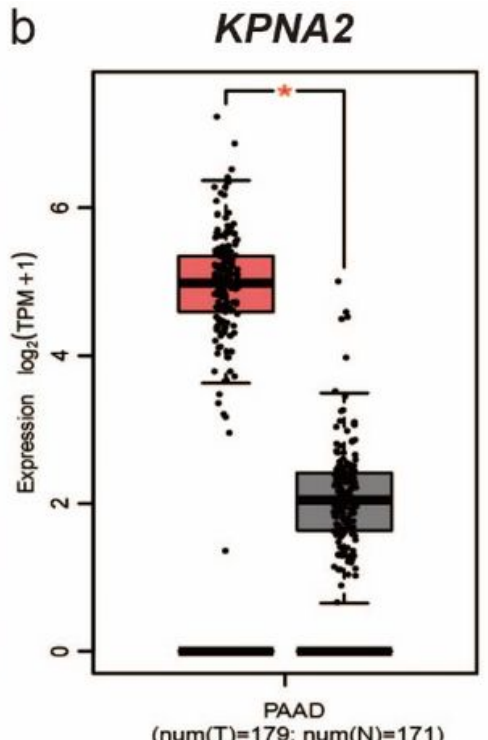

(num(T)=179; num(N)=171)
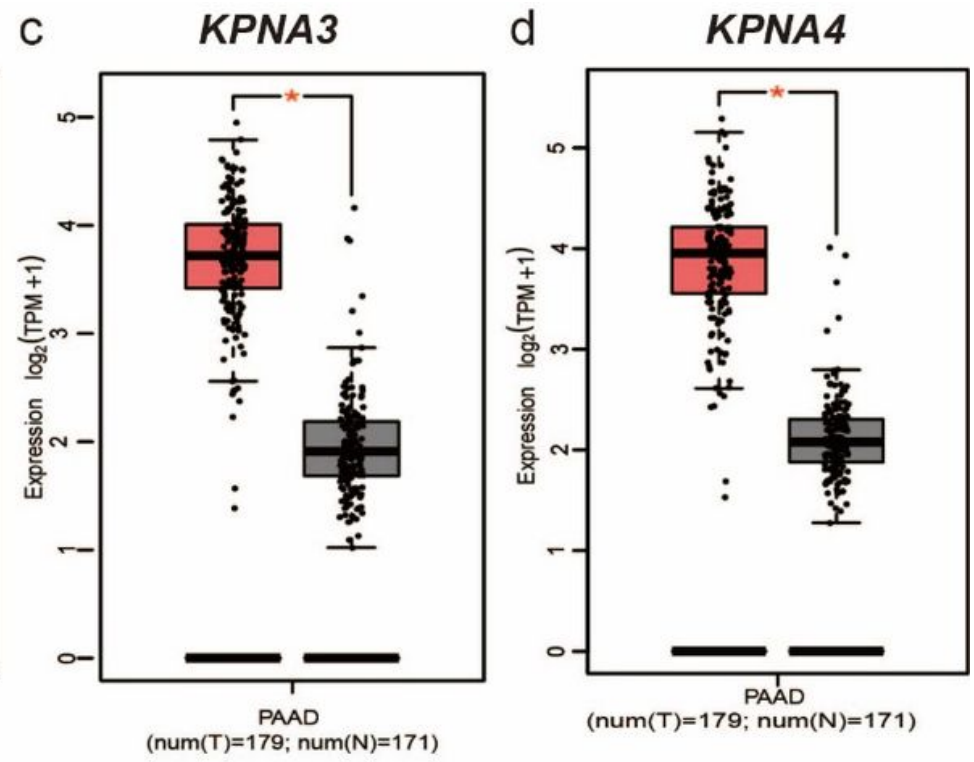

g

KPNA7

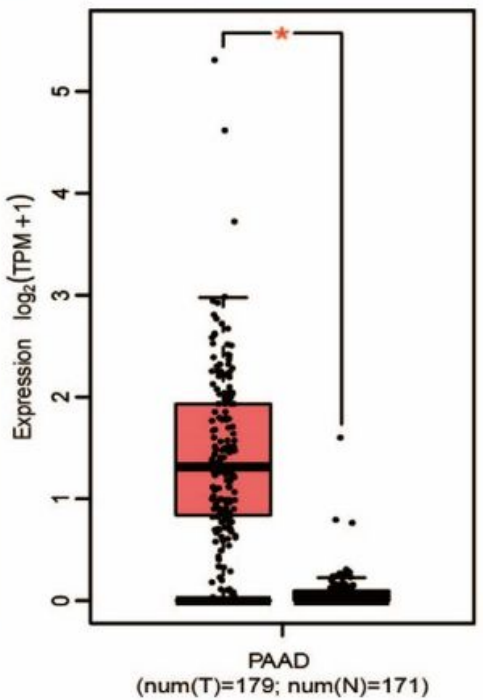

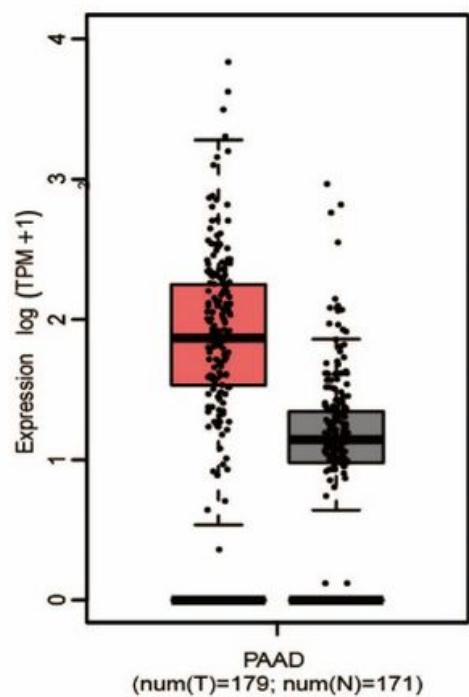

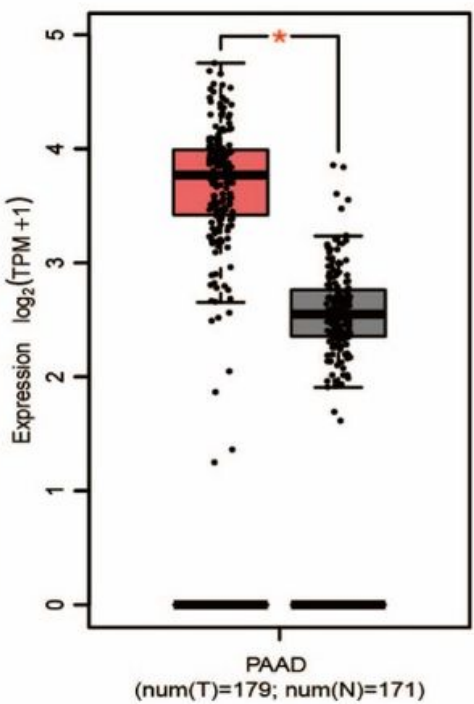

Figure 2

mRNA expression pattern of KPNAs in patients with PAAD (GEPIA2). red: tumor; gray: normal; $p$-value was set at 0.01. * Indicates statistically significant results. 
a

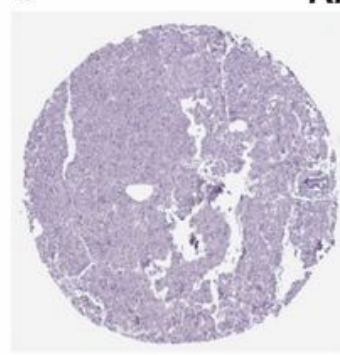

Normal

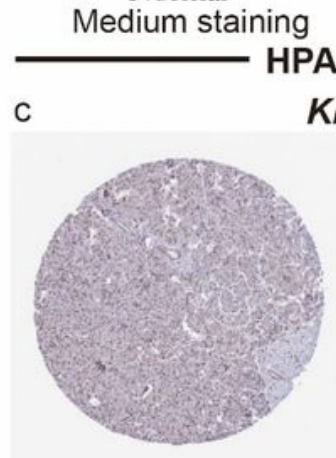

Normal

High staining

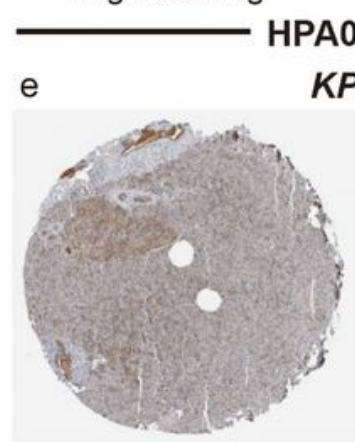

Normal Low staining

g

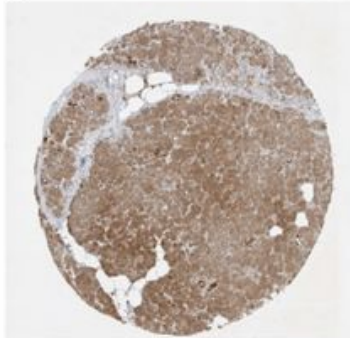

Normal

Medium staining

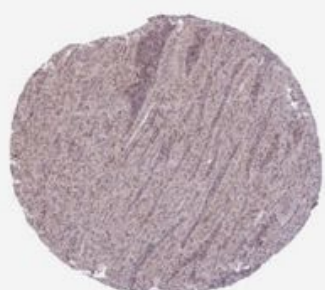

Tumor Medium staining KPNA3

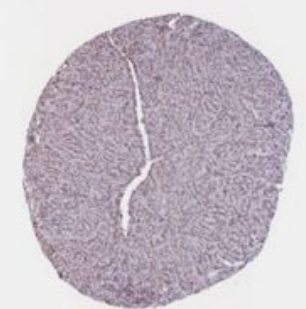

Tumor Medium staining

KPNA5

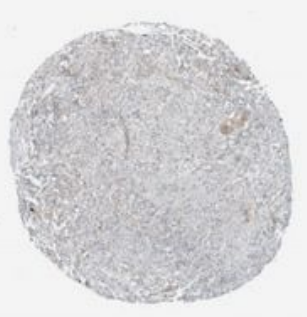

Tumor

Medium staining CAB026464 KPNA7

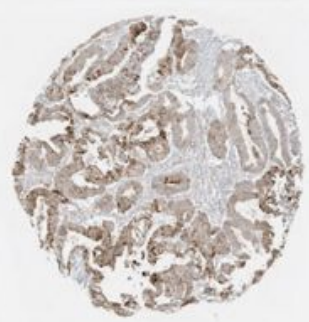

Tumor

High staining

HPA031395

b KPNA2

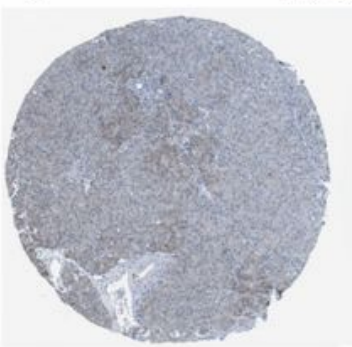

Normal
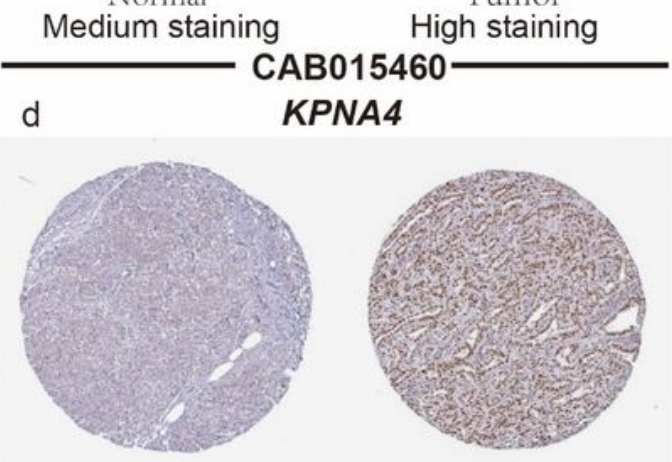

Normal Medium staining
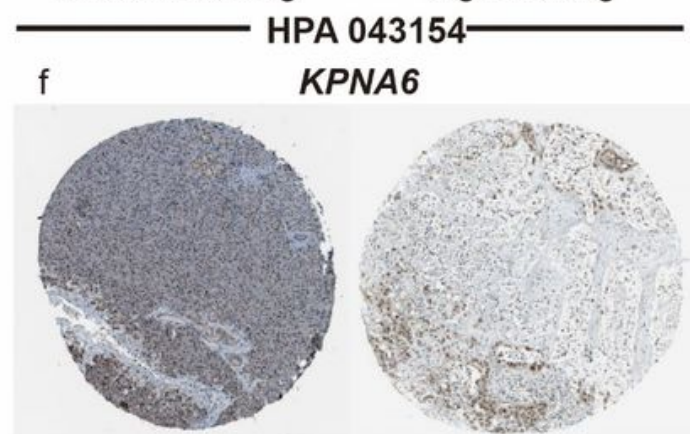

Normal

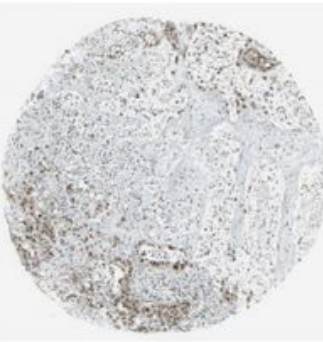
High staining HPA018863

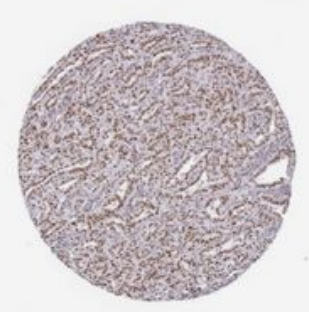

Tumor High staining

Tumor

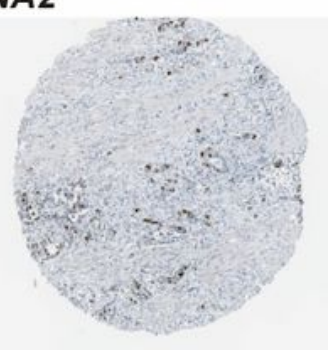

Tumor

h staining

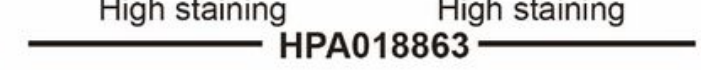

\section{Figure 3}

Representative IHC images of KPNA family members detected in patients with PAAD and normal pancreatic tissues (Human Protein Atlas). 


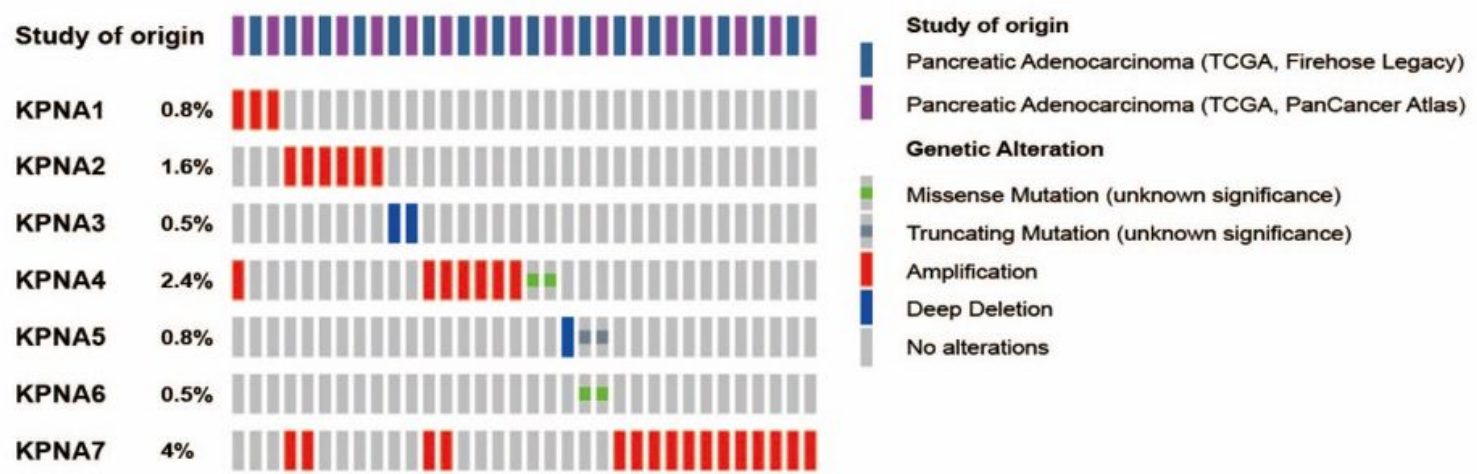

b
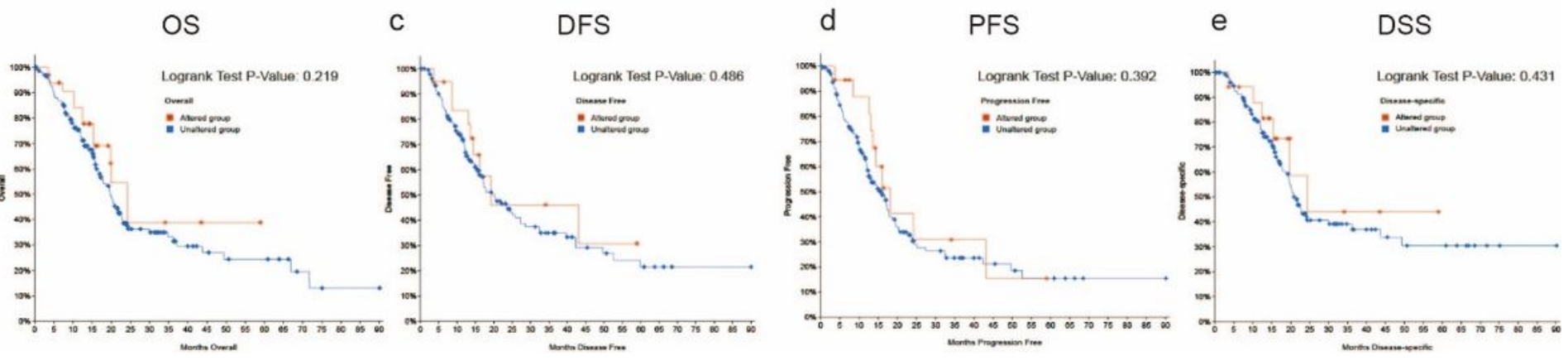

Figure 4

KPNA family gene expression and mutation analysis in pancreatic adenocarcinoma (c-BioPortal). (a) Distribution and proportion of samples with different types of alterations in the KPNA family. The right part of the figure without alterations was not included. (b) Overall survival in cases. (c) Disease-free survival in cases. (d) Progression-free survival in cases. (e) Disease-specific survival in cases. 

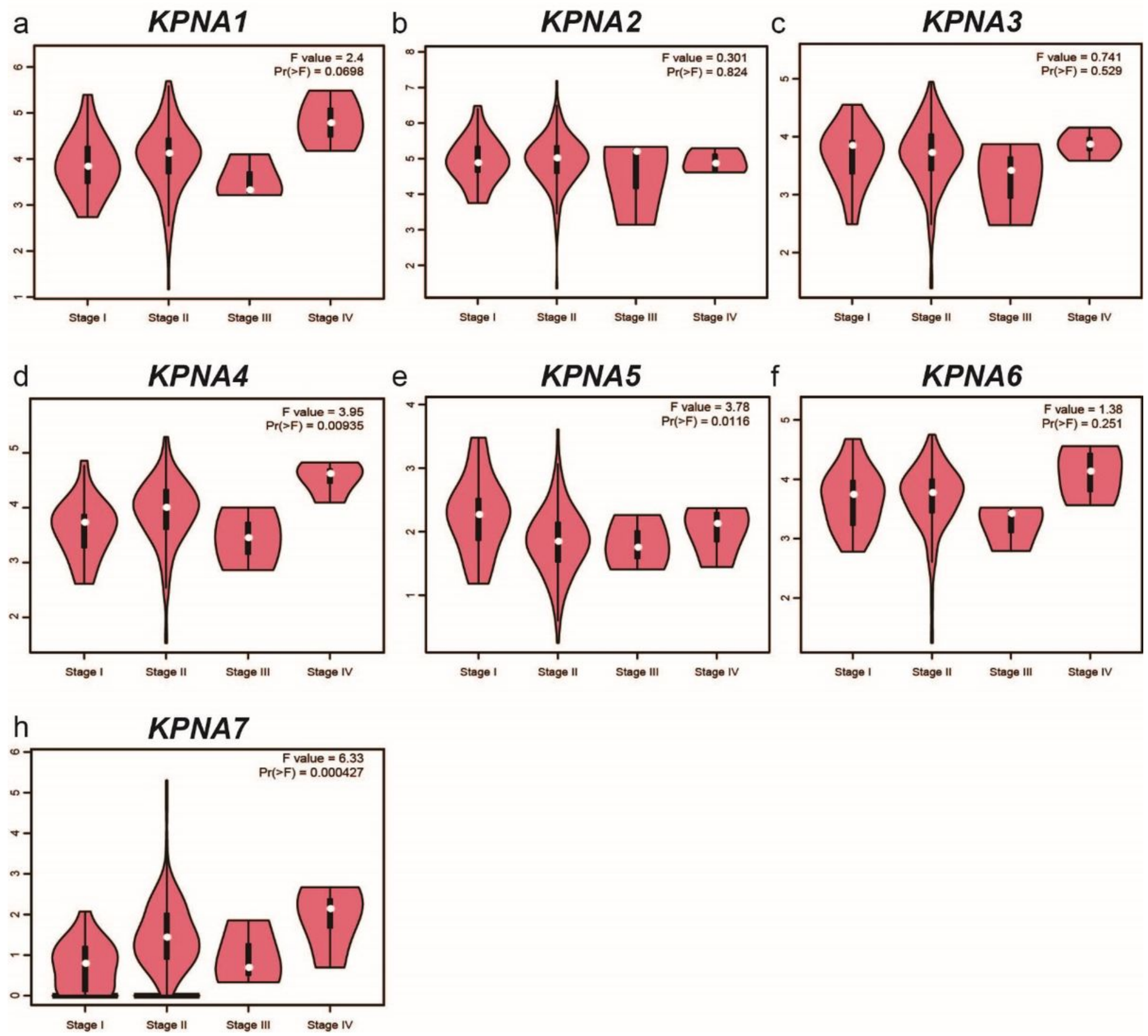

Figure 5

The relationship between KPNAs expression and tumor stage in patients with PAAD (GEPIA2). 


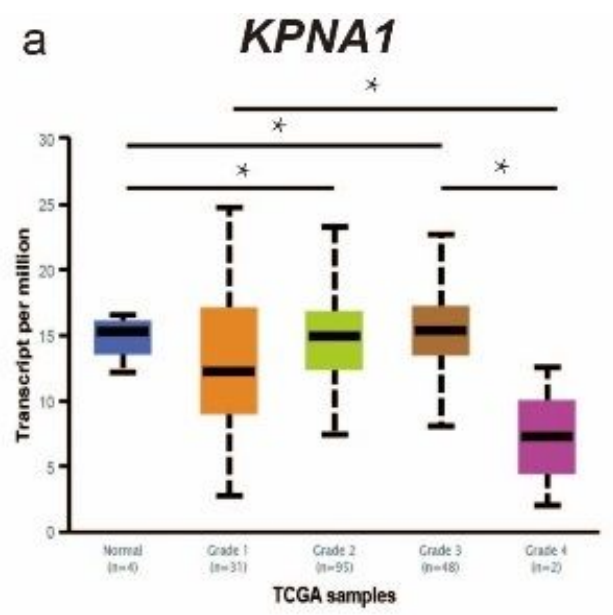

b KPNA2

C

KPNA3

d

KPNA4
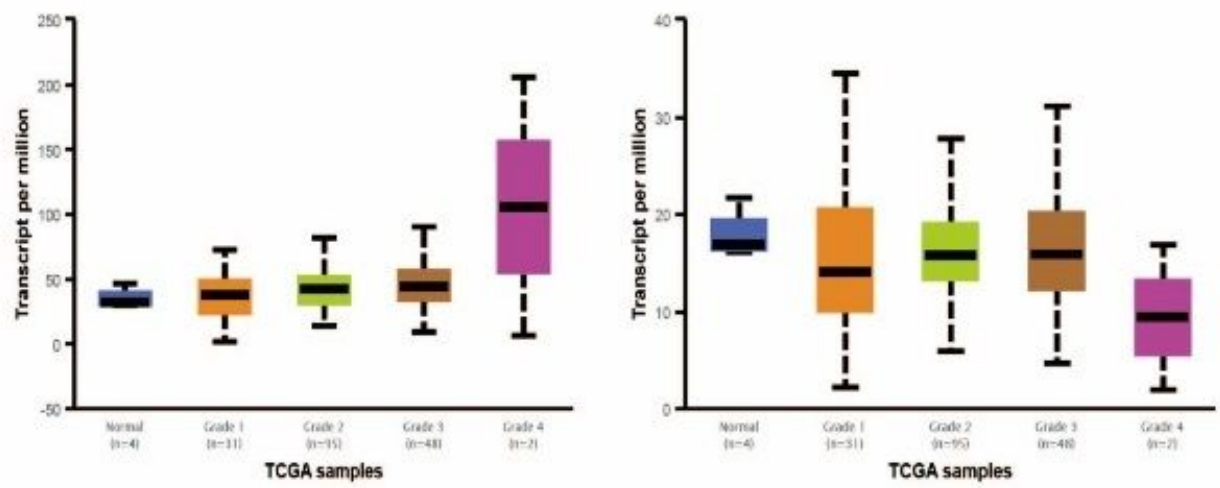

e KPNA5

f

KPNA6
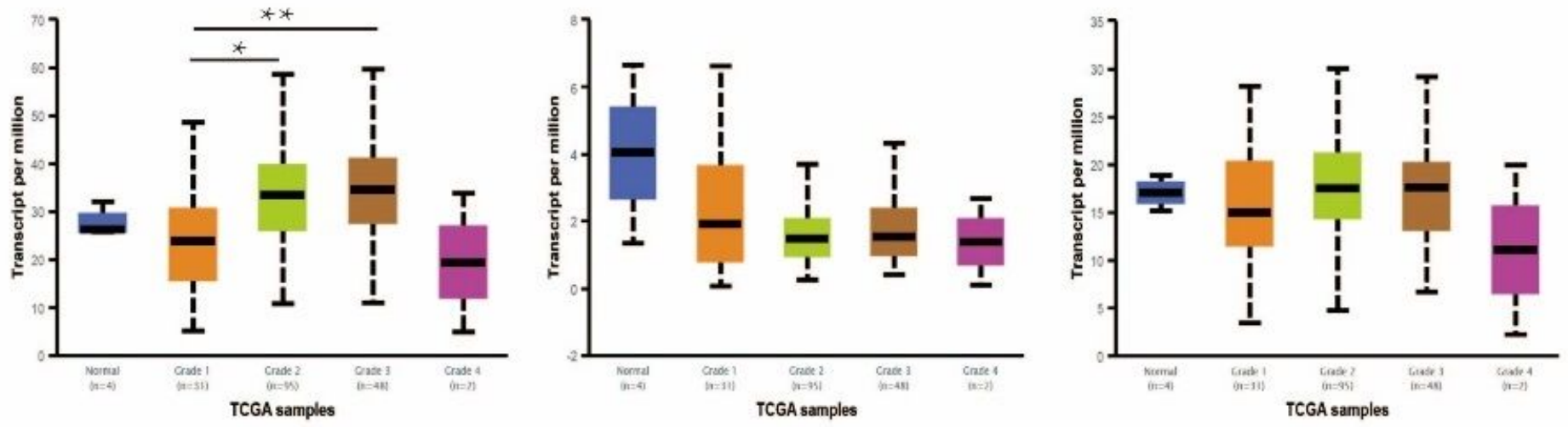

g

KPNA7

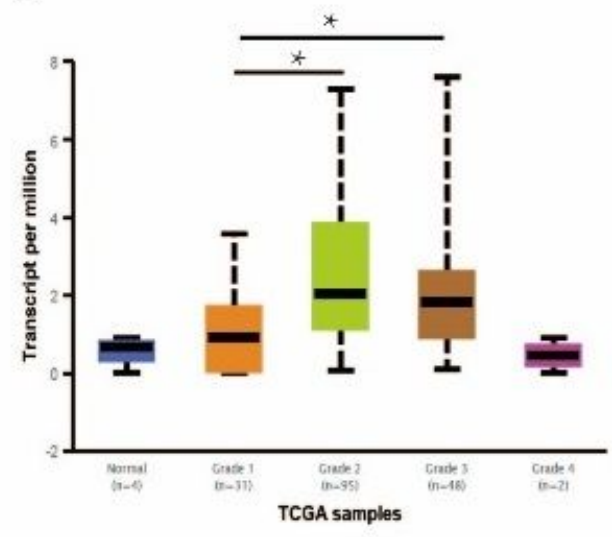

Figure 6

The relationship between KPNA mRNA expression and tumor grade in PAAD patients (UALCAN). * $P<0.05$; $\star \star P<0.01 ; * \star * P<0.001$. 

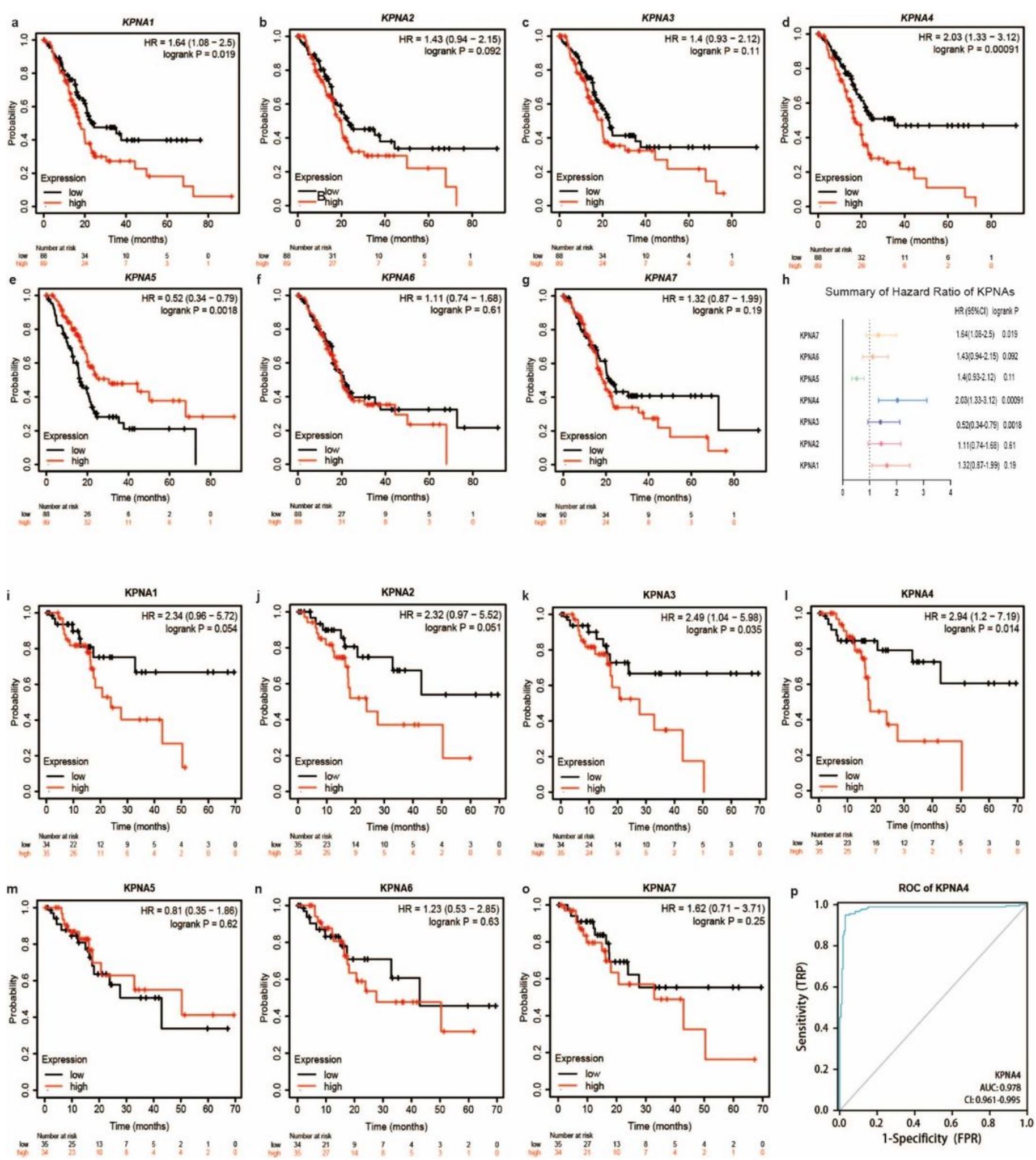

Figure 7

Survival analyses of the KPNA family in patients with PAAD (Kaplan-Meier Plotter). (7a-7g): The overall survival (OS) analyses of KPNAs; KPNA4 and KPNA5 were showed prognostic significance. 7h: The summary of Hazard Ratio of KPNAs (7i-7o): The relapse-free survival (RFS) analyses of KPNAs. KPNA3 and KPNA4 showed prognostic significance. (7p) Receiver Operating Characteristic Curve. AUC of KPNA4 
implies KPNA4 may be a biomarker in patients with PAAD. Abbreviations: PAAD, Pancreatic ductal adenocarcinoma; OS, overall survival; RFS, relapse-free survival; AUC, Aera under the curve.

a

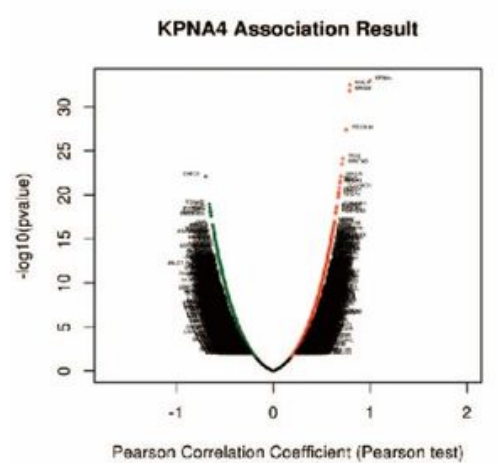

c

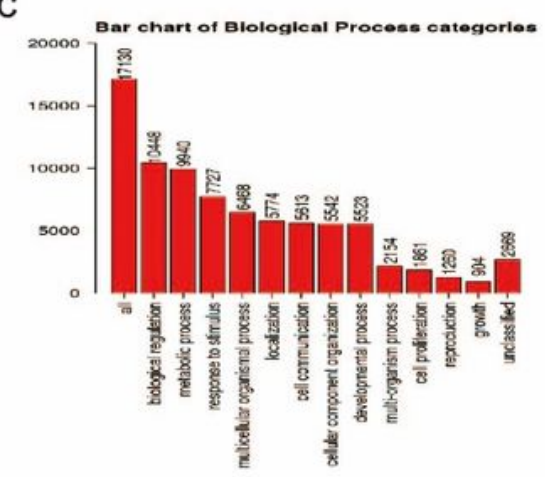

b

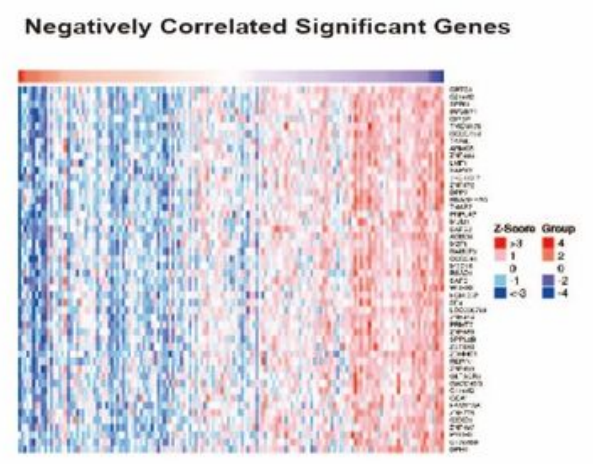

Positively Correlated Significant Genes

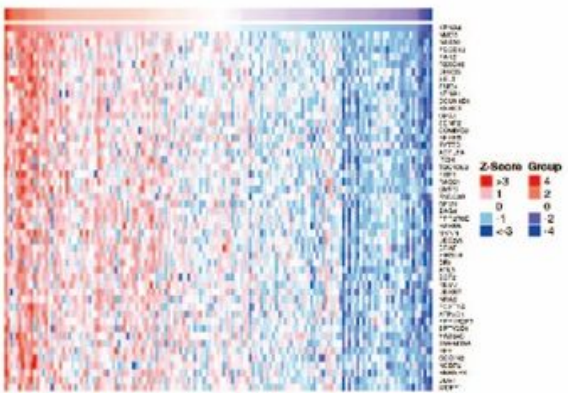

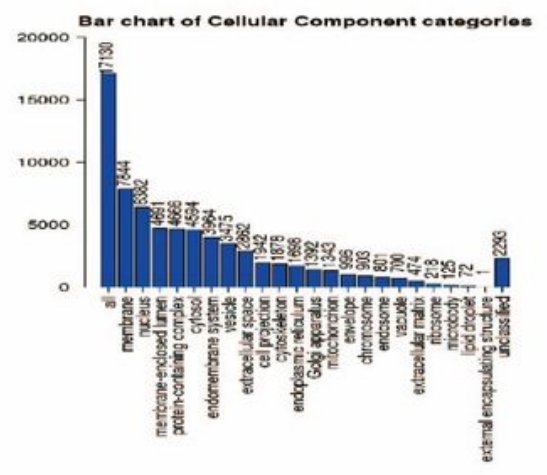

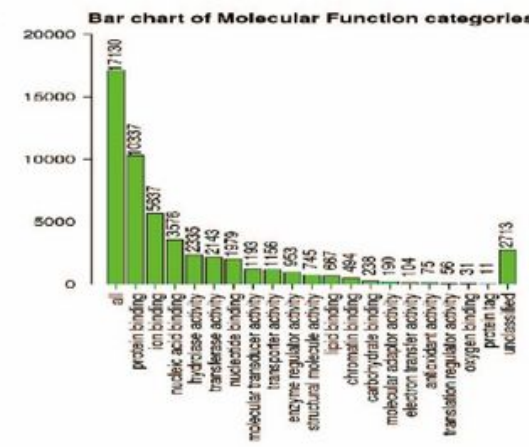

d

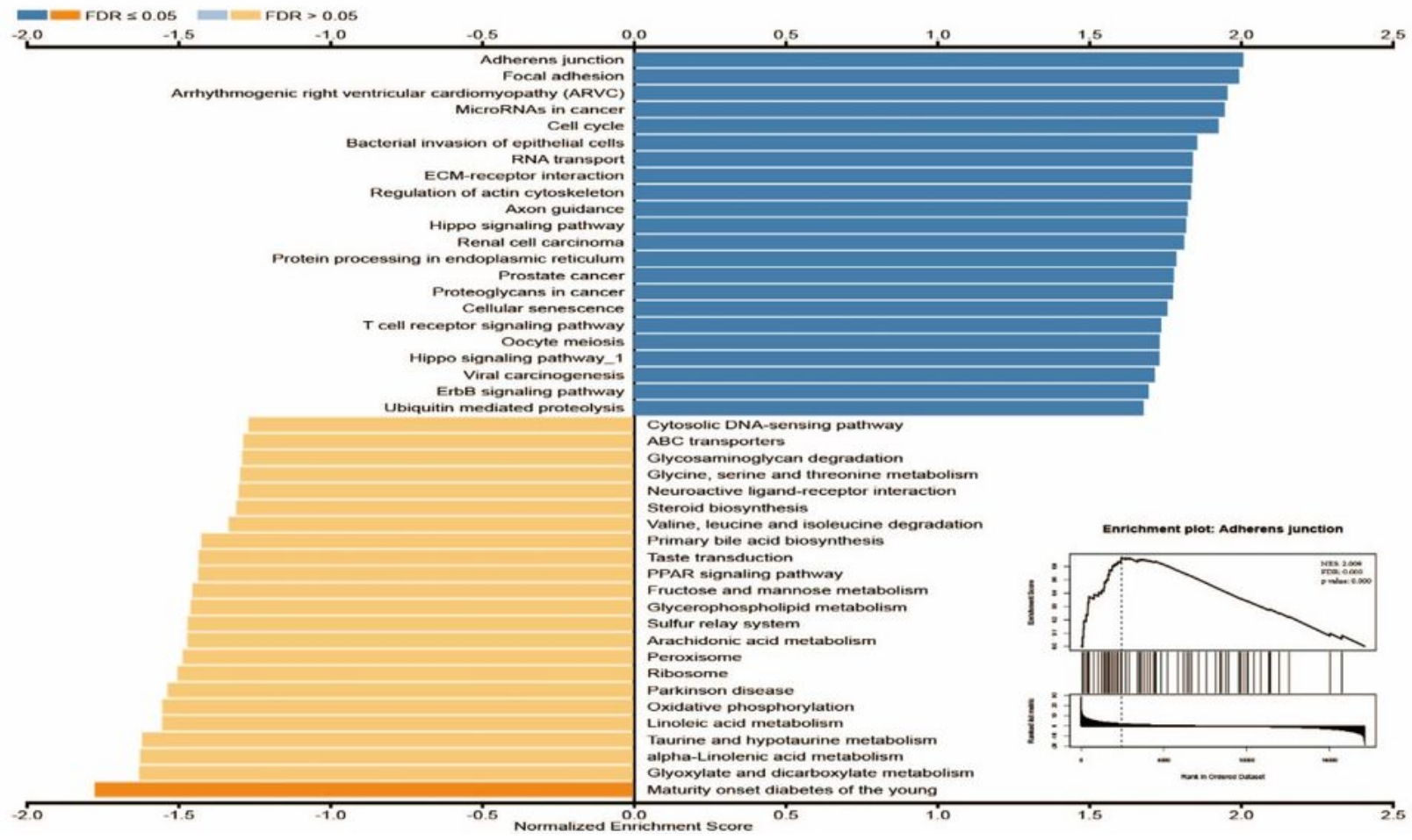

Figure 8

Significantly enriched Gene Ontology (GO) annotations and KEGG pathways of KPNA4 in PAAD cohort (LinkedOmics). (a) Pearson test identifies highly related genes with KPNA4 in PAAD cohort; (b) Heat maps showed positive and negative correlations of the top 50 genes with KPNA4 in PAAD. (c) Gene Ontology 
(GO) functional enrichment analysis. (d) Significantly enriched KEGG pathways of KPNA4 in PAAD cohort.

\section{ADHERENS JUNC TION}

Inside

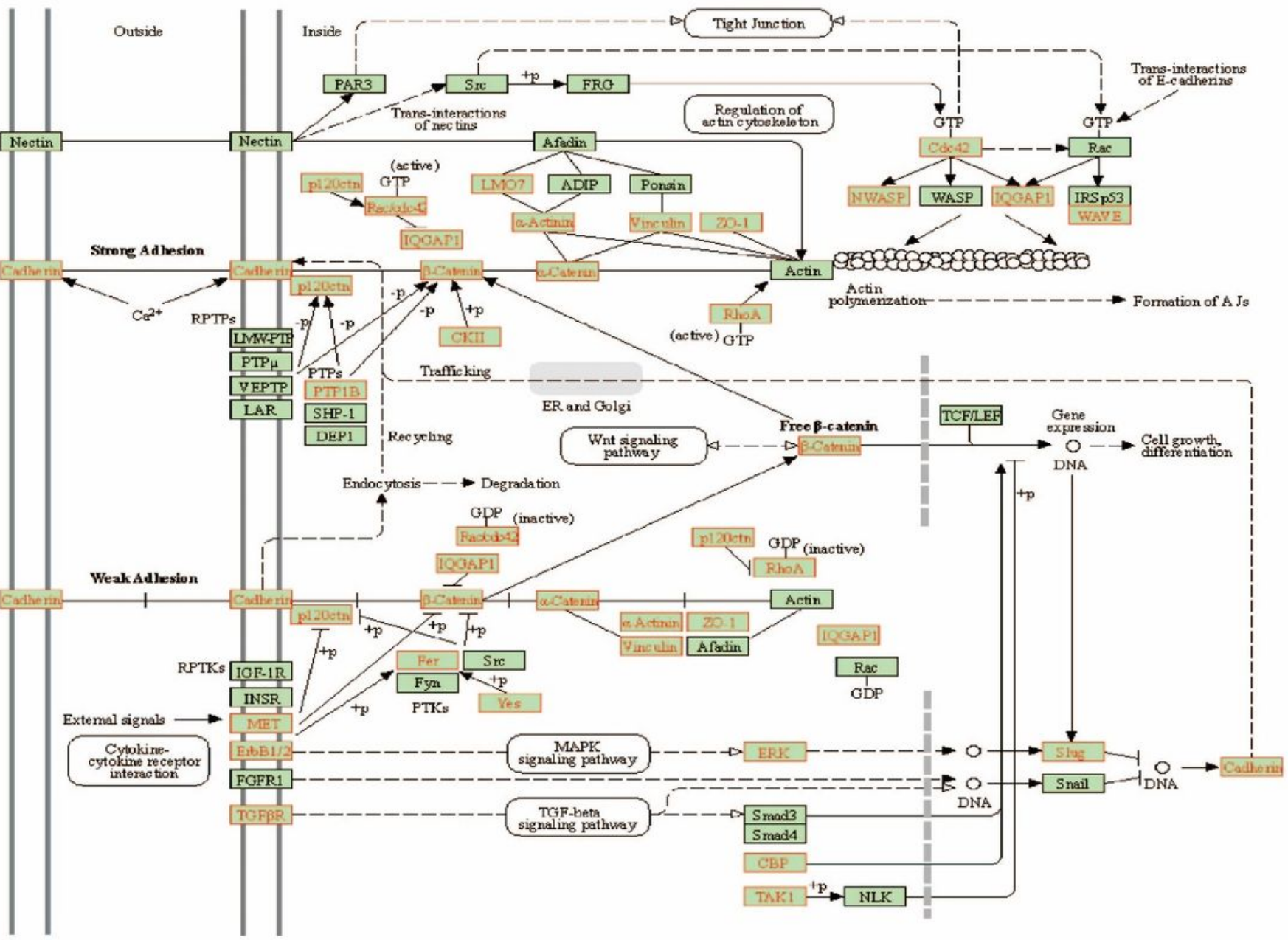

Figure 9

Adherens junction Regulatory pathway by KPNA4 aberration in Pancreatic adenocarcinoma (KEGG). 


\section{genes coexpressed with KPNA4}

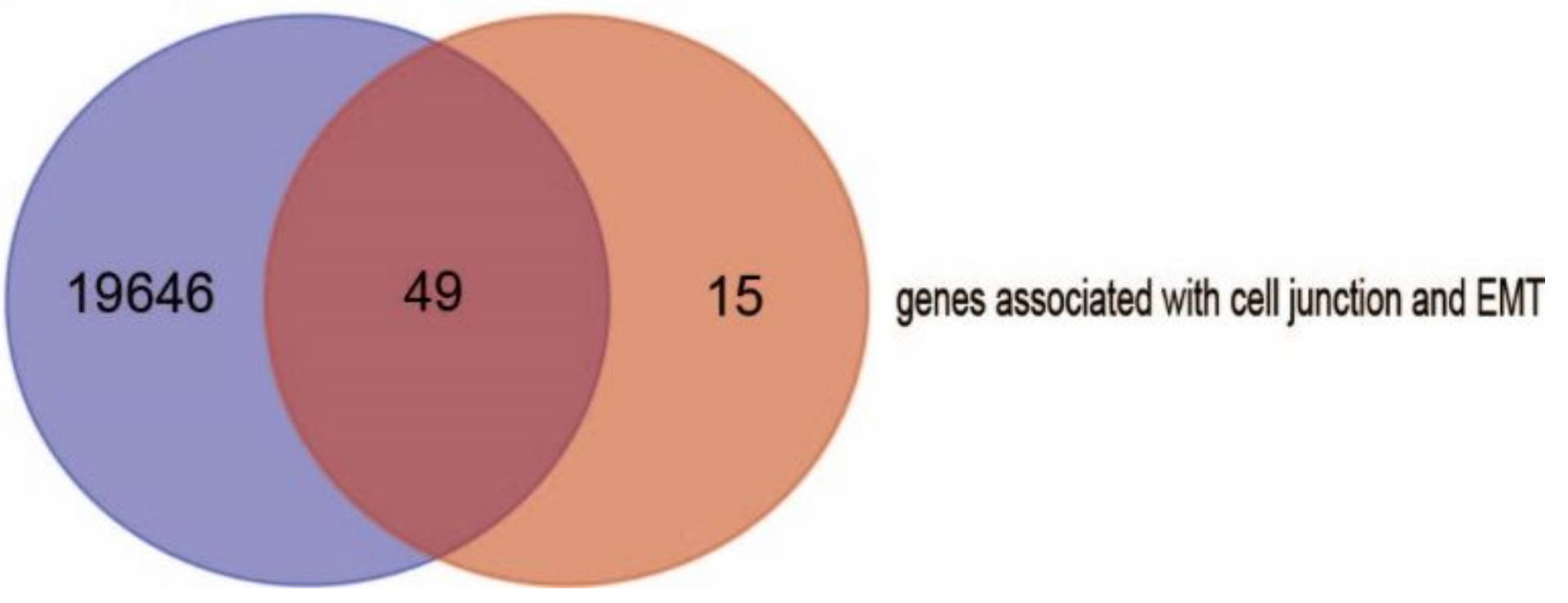

Figure 10

Venn diagram created by the website tool (http://bioinformatics.psb.ugent.be/webtools/Venn) showed the overlapped genes. 
a

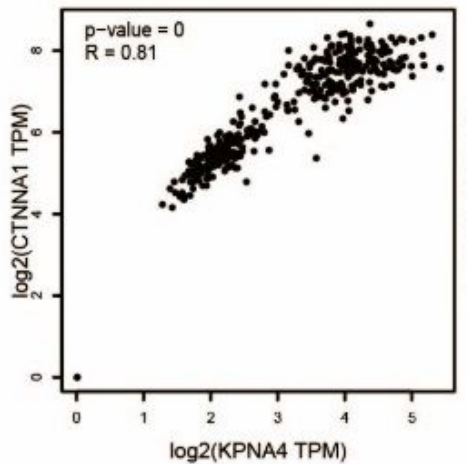

C

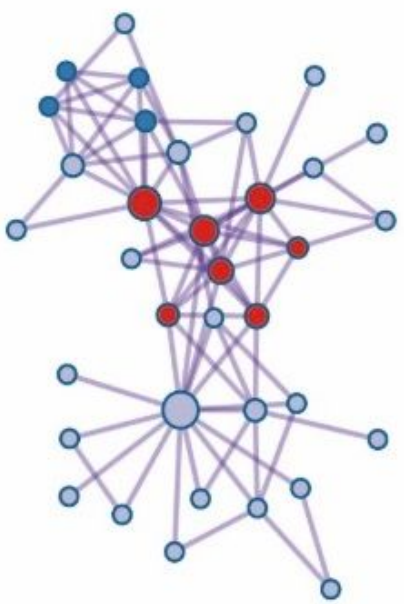

CTNNB1

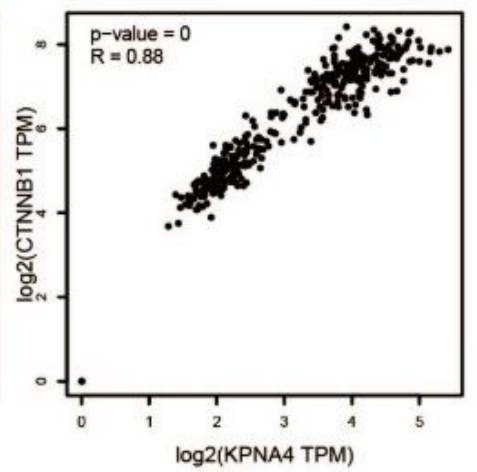

b

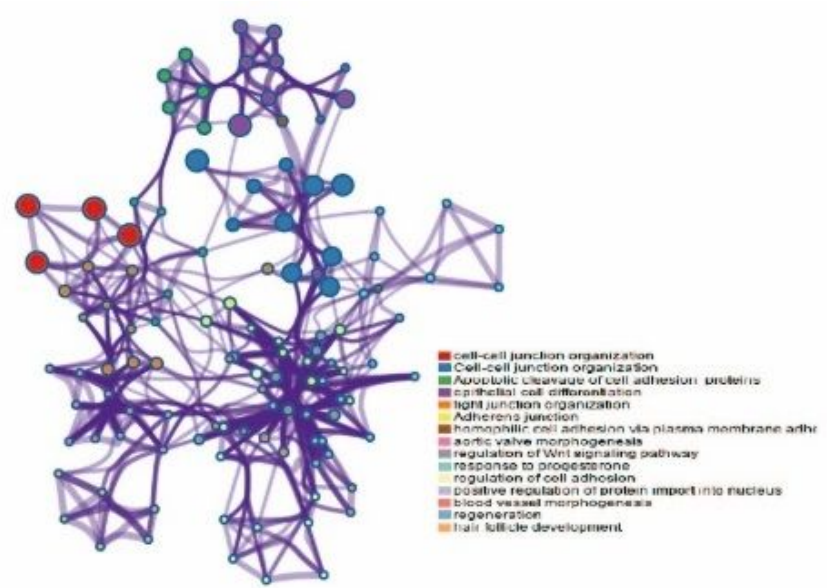

d
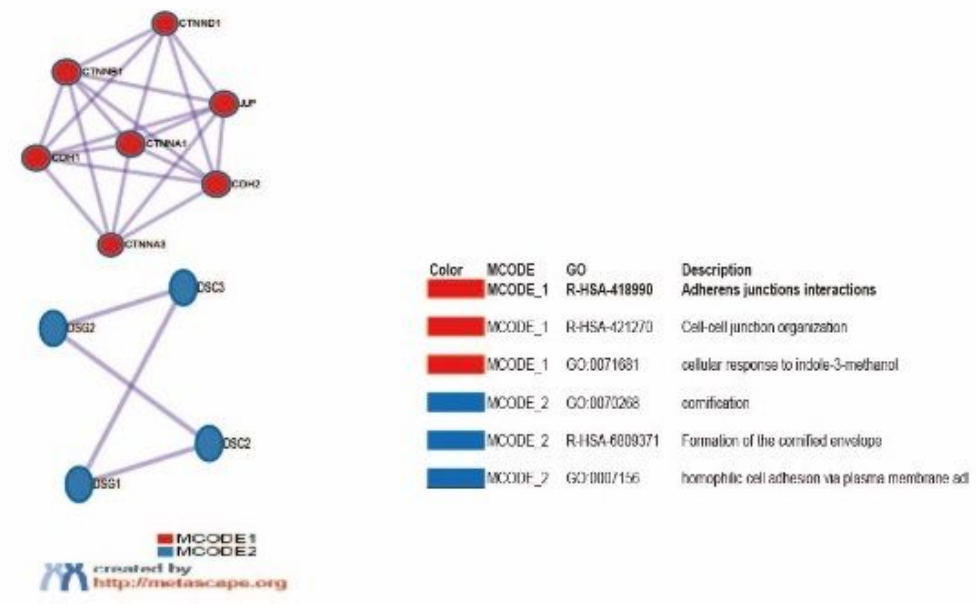

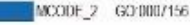

mophes: osal acheson ua piasma memtrane ad

Neoplastic Cell Transformation

Neoplasm Invasiveness

Cardiomyopathy, right ventricular

Mammary Neoplasms

Palmoplantar Keratosis

Tachycardia, Ventricular

Acantholysis

Nikolsky sign

Hypotrichosis

Neoplasm Metastasis

Kinky hair texture

Afro-textured hair

Nappy hair texture

Wooly hair

Fragile skin

Nail abnormality

Nail Diseases

Breast Carcinoma

Stomach Neoplasms

Sudden Cardiac Death

\section{Figure 11}

The correlation between KPNA4 and genes of cell adhesion junction in patients with PAAD. (a) Two genes displayed the strongest linear correlation with KPNA4 in GEPIA2. (b) Gene process and pathway enrichment analysis created by Metascape. Term-rich network: colored by cluster ID, nodes sharing the same cluster ID are usually close to each other. Table pathway enrichment correlated with cell junction organization. (c) Molecular interaction network and MCODE components created by Metascape with KPNA4 correlation genes. (d) Heatmap of enrichment pathway analysis created by Metascape. 